\title{
Multidimensional Assignment Problem for multipartite entity resolution
}

\author{
Alla Kammerdiner • Alexander \\ Semenov • Eduardo L. Pasiliao
}

Received: date / Accepted: date

\begin{abstract}
Multipartite entity resolution aims at integrating records from multiple datasets into one entity. We derive a mathematical formulation for a general class of record linkage problems in multipartite entity resolution across many datasets as a combinatorial optimization problem known as the multidimensional assignment problem. As a motivation for our approach, we illustrate the advantage of multipartite entity resolution over sequential bipartite matching. Because the optimization problem is NP-hard, we apply two heuristic procedures, a Greedy algorithm and very large scale neighborhood search, to solve the assignment problem and find the most likely matching of records from multiple datasets into a single entity. We evaluate and compare the performance of these algorithms and their modifications on synthetically generated data. We perform computational experiments to compare performance of recent heuristic, the very large-scale neighborhood search, with a Greedy algorithm, another heuristic for the MAP, as well as with two versions of genetic algorithm, a general metaheuristic. Importantly, we perform experiments to compare two alternative methods of re-starting the search for the former heuristic, specifically a random-sampling multi-start and a deterministic design-based multi-start. We find evidence that design-based multi-start can be more efficient as the size of databases grow large. In addition, we show that very large scale search, especially its multi-start version, outperforms simple Greedy heuristic. Hybridization of Greedy search with very large scale
\end{abstract}

Alla Kammerdiner

University of Florida Research and Engineering Educational Facility (REEF), Shalimar, FL E-mail: alla.ua@gmail.com

Alexander Semenov

Herbert Wertheim College of Engineering, University of Florida, FL, USA

E-mail: asemenov@ufl.edu

Eduardo L. Pasiliao

Munitions Directorate, Eglin Air Force Base, Eglin, FL

E-mail: elpasiliao@gmail.com 
neighborhood search improves the performance. Using multi-start with as few as three additional runs of very large scale search offers some improvement in the performance of the very large scale search procedure. Last, we propose an approach to evaluating complexity of the very large-scale neighborhood search.

Keywords Multidimensional assignment problem · multipartite entity resolution · Very large scale neighborhood search · Greedy algorithm · genetic algorithm

\section{Introduction}

In this paper, we describe a computationally challenging new application of the multidimensional assignment problem (MAP). We show how this application of the MAP arises naturally when simultaneously merging multiple massive datasets. Because finding global optimum for large-size instances of the MAP remains a significant hurdle, we apply a recent metaheuristic approach, known as the very large-scale neighborhood search (VLSNS), for solving the MAPs. Due to its novelty, the VLSNS algorithm is much less studied than other approaches for the MAP. In particular, it has not been evaluated on larger-sized problem instances, with exception of limited computational experiment in 26]. Therefore, we conduct extensive numerical experiments aimed at comparison of this solution method with state-of-the-art Greedy algorithm and with the global optimal solutions given by a standard linear solver such as Gurobi. We find that this novel metaheuristic shows good performance in our numerical comparison, and therefore, can be used in the context of merging multiple datasets known as multipartite entity resolution.

The amount of the information on the Internet increases every year. In 2005 there were 150 exabytes digital information produced; and there were already 1200 exabytes in 2010 21. Nearly $70 \%$ of this content is user generated [46]. One of the enablers of rapid growth of user-generated content are social media sites. Now there are hundreds of popular social media sites in the Internet. Many individuals maintain accounts at several sites at the same time. Social media sites allow its users to maintain a public profile describing the user. Contents of the profile vary from site to site. Different sites typically concentrate on different aspects of user's identity, and as a result information presented in user profiles is presented in different ways and typically there is not much overlapping. For instance, users of LinkedIn typically provide to the site information regarding their name, education, and employment details. Nickname, name, and such details as location may be found from Twitter, and Instagram keeps photos and details of locations visited by the user; typically the user is identified by her screenname. In order to gain complete information regarding particular user, records from multiple sites should be linked into one entity.

Record linkage problem, a process of linking records representing the same real world entity, arises in many other applications, such as linking descriptions of the same product from multiple e-commerce sites. Another example 
Table 1 Example, three-partite entity matching

\begin{tabular}{|l|l|l|l|l|l|}
\hline $\mathcal{A}-\mathcal{B}$ & $\operatorname{sim}$ & $\mathcal{B}-\mathcal{C}$ & $\operatorname{sim}$ & $\mathcal{A}-\mathcal{C}$ & $\operatorname{sim}$ \\
\hline$\left(a_{1}, b_{1}\right)$ & 0.4 & $\left(b_{1}, c_{1}\right)$ & 1.0 & $\left(a_{1}, c_{1}\right)$ & 1.0 \\
\hline$\left(a_{1}, b_{2}\right)$ & 0.6 & $\left(b_{1}, c_{2}\right)$ & 0.1 & $\left(a_{1}, c_{2}\right)$ & 0.1 \\
\hline$\left(a_{1}, b_{3}\right)$ & 0.6 & $\left(b_{1}, c_{3}\right)$ & 0.1 & $\left(a_{1}, c_{3}\right)$ & 0.1 \\
\hline$\left(a_{2}, b_{1}\right)$ & 0.6 & $\left(b_{2}, c_{1}\right)$ & 0.1 & $\left(a_{2}, c_{1}\right)$ & 0.1 \\
\hline$\left(a_{2}, b_{2}\right)$ & 0.6 & $\left(b_{2}, c_{2}\right)$ & 1.0 & $\left(a_{2}, c_{2}\right)$ & 1.0 \\
\hline$\left(a_{2}, b_{3}\right)$ & 0.6 & $\left(b_{2}, c_{3}\right)$ & 0.1 & $\left(a_{2}, c_{3}\right)$ & 0.1 \\
\hline$\left(a_{3}, b_{1}\right)$ & 0.6 & $\left(b_{3}, c_{1}\right)$ & 0.1 & $\left(a_{3}, c_{1}\right)$ & 0.1 \\
\hline$\left(a_{3}, b_{2}\right)$ & 0.6 & $\left(b_{3}, c_{2}\right)$ & 0.1 & $\left(a_{3}, c_{2}\right)$ & 0.1 \\
\hline$\left(a_{3}, b_{3}\right)$ & 1.0 & $\left(b_{3}, c_{3}\right)$ & 1.0 & $\left(a_{3}, c_{3}\right)$ & 1.0 \\
\hline
\end{tabular}

is consolidating several databases with customers' data when companies are merged. Record linkage problem is challenging, as representation of the same entity may vary across the sources, there may be little or no overlapping in the description, and some sources may contain inaccurate or even conflicting information.

Typical solution for pairwise entity matching is to define scoring function which returns result of pairwise comparison of records, then apply this to all pairs of potential matches, and extract pairs whose scoring is maximal. This problem is modeled in [44] as maximum weighted matching on a bipartite graph. Often scoring function is restricted to be boolean, i.e. it takes values either 1 or 0 if entities match or not, respectively [4. There are methods describing record linkage provided there are erroneous values in some of the records [18. Pairwise record linkage problem seeks to identify the relations between elements of two datasets; this problem may be solved by computing similarity between each pair of items in those datasets, and finding the most similar pairs.

However, this approach would not work for comparison between three and more datasets. This is illustrated by example below.

Example 1 Suppose we have three datasets: $\mathcal{A}=\left\{a_{1}, a_{2}, a_{3}\right\}, \mathcal{B}=\left\{b_{1}, b_{2}, b_{3}\right\}$, $\mathcal{C}=\left\{c_{1}, c_{2}, c_{3}\right\}$. We perform pairwise comparisons between the elements using non-transitive similarity function, and get the results as displayed in Table 1 The goal is to find such matching between elements of $\mathcal{A}, \mathcal{B}$, and $\mathcal{C}$ so that the sum of the scores is maximized. True matching is $\left(a_{1}, b_{1}, c_{1}\right),\left(a_{2}, b_{2}, c_{2}\right)$, $\left(a_{3}, b_{3}, c_{3}\right)$, however, as $\operatorname{sim}\left(a_{1}, b_{1}\right)=0.4$, sequential matching approaches may incorrectly result in matching $\left(a_{2}, b_{1}, c_{1}\right),\left(a_{1}, b_{2}, c_{2}\right),\left(a_{3}, b_{3}, c_{3}\right)$

In the current paper, we study multipartite records linkage problem by formulating it as the multidimensional assignment problem (MAP). Besides the advantage of multipartite entity resolution over sequential bipartite, which was illustrated in Example 1, the formulation of multipartite entity resolution using the MAP can offer an additional advantage in terms of matching precision. In fact, this formulation allows one to establish the most likely linkage of records by solving the MAP to obtain its global optimum. It is noteworthy that although multipartite entity resolution has been discussed in [52, to the best of our knowledge, it has not been formulated as the MAP. The MAP is 
a well-known NP-hard problem in combinatorial optimization. This problem arises in various economic, military, and health care applications, but most notably in the context of multi-sensor fusion and multi-target tracking.

Our main contributions are as follows:

- We formulate multipartite records linkage problem as multidimensional assignment problem (MAP).

- We perform experimental evaluation of novel search algorithm for solving MAP, including the exploration or multi-start phase in this search.

- We experimentally show that our algorithm finds better solution than Greedy algorithm.

- We compare our algorithm results to optimal solutions obtained by genetic algorithms and Gurobi solver.

- Importantly, we compare two distinct multi-start schemes (a random multi-start versus a design-based multi-start) for the exploration phase, and find computational evidence in support of the latter.

The remainder of this paper is organized as follows. Section 2 positions our paper in the related research literature and discusses the key contributions to both operations research and computer science. In Section 3, we first present intuition behind our approach to formulating general record linkage problems for multipartite entity resolution as the MAP, and then we show how the problem statement is mathematically derived. In Section 4 , we outline two algorithms developed specifically for solving the MAP and focus our attention on a newer, VLSN-based approach. For completeness, we include pseudocode of each algorithm. First, we briefly describe Greedy algorithm. Second, we present the VLSN local search algorithm. Third, we propose several multi-start strategies for the VLSN search and discuss the resulting VLSN-based metaheuristics. Section 5 presents our approach to evaluating complexity of the VLSN-based metaheuristics using two related Markov chains. In Section 6, we study the performance of VLSN-based metaheuristics in terms of the solution quality and compare VLSN with non-VLSN algorithms, including Greedy algorithms, standard metaheuristic such as genetic algorithm, and an all-purpose solver such as Gurobi. Additionally, we illustrate the application of our approach to real record linkage and multipartite resolution on synthetic dataset. Finally, Section 7 concludes.

\section{Related research}

The paper builds upon several strands of the literature in mathematical programming and computer science. On one side, the paper is concerned with the multidimensional assignment problem in combinatorial optimization. On the other side, the study advances record linkage, entity resolution, and other related areas in relational databases. In this section, we review the prior research in these areas, starting first with computer science and then continuing with optimization. 
In computer science, related areas include research on record linkage, network alignment, user identity reconciliation, de-anonymization of online social networks, entity resolution, and reference reconciliation. Survey [5] reports entity resolution and record linkage methods used in relational databases. The survey focuses on criteria for entity matching (such as exact match, distance match, etc); survey considers pair-wise linkage only. Algorithm for linking user accounts across two social media sites is presented in the article 44. Authors model results produced by users at different sites as trajectories (these trajectories are modeled as buckets of records), compute the scores for every pair of users, represent this data as bipartite graph and find the maximum weighted matching on this graph. Authors consider linking across two sites. Review of user identity linkage across social networks [4] concentrates on linkage of user profiles between two social networks (source site and target site). Linking users between two social media sites by comparing usernames is presented in [1]. Authors of [20] construct relationship between two social networks by applying decision tree classification to set of users' features (same highschool, etc).

According to [36], entity resolution (identifying entities, that represent the same real-world object) has quadratic complexity, and because of that many algorithms employ blocking: cluster similar elements into blocks, and then perform cluster-wise comparison. Paper presents comprehensive survey of blocking methods; it focuses on resolution of entities over two datasets. Evaluation of entity resolution approaches is presented in 32 . Authors consider real world datasets, and several algorithms for evaluation, however matching is carried out between two datasets only. Linking temporal entities is described in [34]. Paper concentrates on building temporal model of user profile evolution, and then provides matching algorithm across two datasets. Paper [45] describes how fuzzy clustering may be used as a blocking method; authors applied the entity resolution methods to the repository of Holocaust-era information.

Paper [52] describes entity resolution problem as combinatorial optimization for problem of max-weight graph matching on multi-partite graphs. Paper presents optimization model and several algorithms to solve it. Multipartite entity resolution problem is also studied in [51. Online version of entity resolution with an oracle which correctly labels matching and non-matching pairs through queries is studied in [12. Distributed algorithms for data deduplication are studied in 8]. 41] describes how trackers track user in the Internet and further apply the information for personalized advertising. Papers [17, [50 describe application of crowdsourcing to entity matching. Paper focuses on application of blocking, and minimization of number of comparisons. Identification of Facebook users in Twitter is presented in [23. Paper [54] describes how data on adjacent users in the social network may be used for matching of user profiles between two social networks. Alignment of multiple social networks is studied in 53 . Survey of automated algorithms for duplicate detection and various similarity measures is presented in [11. Authors of [4] view record comparison functions and black-boxes, and analyze generic approach for entity resolution. Authors propose monotonicity conditions of merge, and describe 
entity resolution approach with idempotence, commutativity, associativity and representativity properties.

In combinatorial optimization, the multidimensional assignment problem (MAP), sometimes called the multi-index assignment, was first introduced in 38, 39. This problem belongs to a general class of assignment problems 7 6], such as the linear assignment problem (LAP) and the quadratic assignment problem (QAP). The MAP is shown to be NP-complete [31,14]. In its simplest form, the MAP is usually characterized by two parameters, namely cardinality $N$ and dimensionality $M$. Then the number of feasible solutions of the MAP is $(N !)^{M-1}$, growing super exponentially. Three-dimensional version of the MAP is known as the axial 3-dimensional assignment or axial 3-AP. The axial 3-AP has an alternative closely related problem, known as the planar 3-AP.

The MAP found applications in diverse areas ranging from defense to physics. For example, the axial 3-AP can arise in the context of capital investment, dynamic facility location, satellite launching 38,39. Furthermore, the 3-AP can arise in production planning in automated manufacturing, such as in assembly of printed circuit boards 9, 10. An interesting special case of the axial 3-AP with binary coefficients (i.e., zeros and ones) arises in application to perishable production planning [2]. Applications of the related planar 3-AP include school timetabling 22, 13, experimental design [22, rostering [15, 16, and launch of communication satellites 3 .

The applications discussed so far lead to three-dimension versions of the MAP. A general (axial) MAP with higher dimensionality (i.e., $M>3$ ) can arise in relation to defense and military surveillance application in the field of multi-sensor multiple target tracking [40. In this setting, the task is to solve a data association problem of identifying which sensor measurements or scans belong to which target paths or tracks. The axial MAP with dimensionality of $M=5$ arises in tracking charged elementary particles in colliding beam experiments in high energy physics 43,42. Routing in meshes or two-dimensional array of nodes (i.e., switches at a circuit level or processing elements at network interconnection level) can be formalized as an axial 3-AP with additional side constraints, which can be further reformulated into a standard sparse MAP with dimensionality $M=5$. Recently, biomedical and healthcare application of general MAP to detection and situational assessment of falls and near falls in elderly population using wearable sensors is described and studied in 24, 28 .

In this paper, we contribute to this literature by introducing a new application to record linkage and entity resolution problems in multiple relational databases. The feature of the MAPs arising in this context is that a problem instance has massive cardinality $N$ and small-to-moderate dimensionality $M \in[3,10]$. Because the MAP is generally NP-hard for $M \geq 3$, a natural question is how to solve the MAP instances to perform record linkage of real-life databases. Although we use a standard linear solver to get the global optimal solution, for sufficiently large $N$ even the best solver (e.g., Gurobi) will fail to return the global solution in a reasonable time. Therefore, we examine two approximate algorithm, namely (i) a promising new metaheuristic approach 
(VLSN search) that exploits a way to quickly search through a massive neighborhood of $N$ ! solutions, and (ii) a Greedy heuristic that has good theoretical performance guarantees for asymptotic case $(N \rightarrow \infty)$. Moreover, we hybridize these two heuristics by using a Greedy solution as a starting solution in the VLSN search. Furthermore, we contribute to the literature on search-based metaheuristics by exploring two alternative approaches to sampling or constructing solutions for re-initializing the search in each exploitation stages. These two alternative ways of exploration are random sampling and (deterministic) "design"-based constructive heuristic. Additionally, we contribute to the literature on metaheuristics for the MAP by comparing the VLSN-based algorithms with two versions of genetic algorithm (GA). Our results demonstrate that VLSN search tends to outperform GA on moderate-to-large instances. Last, because little is known about complexity of VLSN search, we propose an approach for evaluating worst-case complexity of the VLSN metaheuristics.

\section{Assignment Formulation of General Record Linkage Problems}

In this section, we formulate a general class of record linkage problems in the context of multipartite entity resolution as the multidimensional assignment problem (MAP), building upon the work of 552 . We discuss variations on this formulation in the conclusion of this section.

To clarify the problem statement, we first present general intuition behind formulation of multipartite record linkage as the MAP. Recall that multipartite record linkage problem arises when merging records in multiple datasets, where each of these datasets contains different aspects of underlying entities. In application to user profile matching, linkage problem is matching records from multiple social media sites (e.g., Google, LinkedIn, Twitter) to associate these records with a user (i.e., entity). To formulate the problem, we assume that media site records have been deduplicated so that each record is unique. Next, we define a similarity function on the $M$-dimensional vector of records where each dimension corresponds to a given social media site (i.e., a given dataset included in a merge). The task is to partition the merged dataset into the $M$-dimensional vectors of records. For example, suppose the record linkage is performed with three records of Google accounts $g_{1}, g_{2}, g_{3}$, two LinkedIn accounts (or records) $l_{1}, l_{2}$, and two Twitter accounts (or records) $t_{1}, t_{2}$. Using similarities among accounts, the most likely matching returns three user profile vectors, namely user profile 1 with $\left(g_{1}, l_{2}, \cdot\right)$, profile 2 with $\left(g_{2}, \cdot, t_{2}\right)$ and profile 3 with $\left(g_{3}, l_{1}, t_{1}\right)$, where represents an empty record or an absence of an account on a given site. The key intuition behind our approach is that the records from $M$ sites are partitioned into the $M$-dimensional vectors or tuples so as to maximize the likelihood of similar records across sites being assigned together into a tuple. Observe that in partition, at most one record from each site is assigned to a given tuple.

Let $E \in \mathcal{E}$ be a real-world entity belonging to set of entities $\mathcal{E}$. Entities are represented by records in a source or a dataset. Entities are not observed 
directly. They are represented by their correct association of records from multiple sources. We assume that if an entity exists in a source then the entity is uniquely represented by a record. In other words, records in each source or dataset are deduplicated. Let a common set $\mathcal{R}$ of all possible records $R$ consist of representations of an entity $E \in \mathcal{E}$ in some source or dataset $D$. Alternatively, for any record $R$ found in some source $D$, we have $R \in \mathcal{R}$.

Let $M$ be the total number of sources or datasets that must be integrated. This integration is done by matching the records of each source or dataset with the corresponding records that represent the same unknown entity in other sources. $M$ is an integer such that $M \geq 2$. For each $k=1,2, \ldots, M$, let $D(k)$ denote a set that consists of a dummy or missing records and $N_{k}$ actual records (which are unique or deduplicated in the $k$-th dataset). Suppose that non-negative integers $i_{k}=0,1, \ldots, N_{k}$ denote the indexes of records in the $D(k)$-th dataset (or source) with the index $i_{k}=0$ representing the missing (or dummy) record, which has no field entries.

Let $\operatorname{sim}(\cdot, \cdot): \mathcal{R} \times \mathcal{R} \mapsto[0,1]$ be a similarity function that compares any pair of records $R_{1}, R_{2} \in \mathcal{R}$, mapping them to their similarity with dissimilar records represented by 0 and similar ones denoted by 1 .

Definition 1 Given datasets $D(1), D(2), \ldots, D(M), M$ corresponding records $R_{1}, \ldots, R_{M}$, and a pair-wise similarity function $\operatorname{sim}(\cdot, \cdot): \mathcal{R} \times \mathcal{R} \mapsto[0,1]$, we define a multi-record similarity function $\operatorname{msim}(\cdot, \ldots, \cdot): \mathcal{R}^{M} \mapsto[0,1]$ as follows:

$$
m \operatorname{sim}\left(R_{1}, \ldots, R_{M}\right)=\sum_{i, j: i<j} \operatorname{sim}\left(R_{i}, R_{j}\right) .
$$

In addition, let us consider a related function $\delta$ :

$$
\begin{aligned}
\delta\left(R_{1}, \ldots, R_{M}\right) & :=\sum_{i, j: i<j}\left(1-\operatorname{sim}\left(R_{i}, R_{j}\right)\right) \\
& =(M-1) M / 2-m \operatorname{sim}\left(R_{1}, \ldots, R_{M}\right) .
\end{aligned}
$$

Furthermore, let $d_{i_{k}}^{k}$ represent the $i_{k}$-th record in the $k$-th dataset for some $k=1,2, \ldots, M$ and $i_{k}=0,1, \ldots, N_{k}$. Then for any given $k, d_{0}^{k}$ is the missing (i.e., dummy) record. Also

$$
D(k)=\cup_{i_{k}=0}^{N_{k}}\left\{d_{i_{k}}^{k}\right\}, \text { for any } k=1, \ldots, M .
$$

We combine $M$ sets $D(1), D(2), \ldots, D(M)$ of entities into the cumulative collection of $M$ datasets, written as

$$
D^{M}:=\{D(1), \ldots, D(M)\}=\cup_{k=1}^{M} D(k) .
$$

Problem Definition. In a general multipartite entity resolution, the goal is to link records from multiple sources (datasets) that corresponds to the same unknown entity. Hence, the decision making problem is to find the most likely partition of all possible $M$-tuples from the cumulative dataset $D^{M}$ into linkages (or $M$-partite matches) and non-linkages, given a collection of $M$ databases. 
We define a linkage (or $M$-partite matching) of records $D_{i_{1} \ldots i_{M}}$ as

$$
D_{i_{1} \ldots i_{M}}:=\left\{d_{i_{1}}^{1}, \ldots, d_{i_{M}}^{M}\right\},
$$

where exactly one record $d_{i_{k}}^{k}$ is included from the dataset $D(k)$ for each $k=$ $1, \ldots, M$. According to this definition, we have $D_{0 \ldots 0}:=\left\{d_{0}^{1}, \ldots, d_{0}^{M}\right\}$.

Next, we define a set $\Gamma$ of all feasible partitions $\gamma$ of $D^{M}$ that satisfy the following conditions (or constraints):

$$
\begin{aligned}
\gamma & =\left\{\gamma_{1}, \ldots, \gamma_{m(\gamma)}\right\} \\
\gamma_{i} \cap \gamma_{j} & \subset D_{0 \ldots 0}, \text { for } i \neq j \\
D^{M} & =\left\{d_{0}^{1}, \ldots, d_{0}^{M}\right\} \cup\left(\cup_{j=1}^{m(\gamma)} \gamma_{j}\right) \\
\gamma_{i} & \neq\left\{d_{0}^{1}, \ldots, d_{0}^{M}\right\} \forall j=1, \ldots, m(\gamma) \\
\forall \gamma_{j}: \gamma_{j} & =D_{i_{1} \ldots i_{M}} \exists ! M \text {-tuple }\left(i_{1}, \ldots, i_{M}\right)
\end{aligned}
$$

These conditions have the following meanings. Eq. 6 states that each feasible partition $\gamma$ consists of $m(\gamma)$ record linkages $\gamma_{i}$ that match records among $M$ datasets. Eq. 7 implies that any two distinct linkages only share missing records. Eq. 8 says that the cumulative collection $D^{M}$ of $M$ datasets or sources is comprised of record linkages forming a partition and missing records from each source. Eq. 9 states that no linkage matches only missing records. Importantly, Eq. 10 guarantees an existence of a unique ordered set of the positions $i_{m}$ of records from each source $m=1, \ldots, M$ for each linkage or matching $\gamma_{j}, j=1, \ldots, m(\gamma)$ of records from a partition $\gamma$. Intuitively, together the conditions insure that a partition covers all the combined data from $M$ sources by splitting these data into the $M$-tuples $\left(i_{1}, \ldots, i_{M}\right)$ of records, which represent a unique hypothesized entity, and that $M$-tuples are distinct up to missing $\operatorname{record}(\mathrm{s})$ for some sources.

A missing record $d_{i_{k}}^{k}$ is included in the above definition, and we denote it by $D_{0 \ldots 0 i_{k} 0 \ldots 0}$. Using $(5)-(10)$, we replace $\gamma_{i}$ 's with the respective linkage variables $D_{i_{1} \ldots i_{M}}$ to rewrite the conditions satisfied by the set $\Gamma$ of all feasible partitions $\gamma$ of $D^{M}$ as follows:

$$
\begin{aligned}
& \gamma=\left\{D_{i_{1} \ldots i_{M}} \text { meets } 12-14\right. \text {, } \\
& \text { for } \left.i_{k}=0, \ldots, N_{k}, k=1, \ldots, M\right\} \\
& D_{i_{1} \ldots i_{M}} \cap D_{j_{1} \ldots j_{M}} \subset D_{0 \ldots 0}, \\
& \text { when } \exists k \in\{1, \ldots, M\}: i_{k} \neq j_{k} \\
& D^{M}=D_{0 \ldots 0} \cup\left(\bigcup_{D_{i_{1} \ldots i_{M}} \in \gamma} D_{i_{1} \ldots i_{M}}\right) \\
& D_{0 \ldots 0} \notin \gamma
\end{aligned}
$$

The above conditions $\sqrt{12}$ and $\sqrt{13}$ are equivalent to the condition that each actual record $d_{i_{k}}^{k}$ is included in only one record linkage $D_{i_{1} \ldots i_{M}} \in \gamma$. We 
formalize this condition by introducing the $0-1$ decision variables:

$$
x_{i_{1} \ldots i_{M}}=\left\{\begin{array}{l}
1, \text { if } D_{i_{1} \ldots i_{M}} \in \gamma \\
0, \quad \text { otherwise }
\end{array}\right.
$$

This leads to the following equivalent characterization of the feasible partitions in $\Gamma$ in terms of the new variables:

$$
\begin{aligned}
& \sum_{\left(i_{1}, \ldots, i_{k-1}, i_{k+1}, \ldots, i_{M}\right)=(0, \ldots, 0)}^{\left(N_{1}, \ldots, N_{k-1}, N_{k+1}, \ldots, N_{M}\right)} x_{i_{1} \ldots i_{M}}=1, \\
& \quad \text { for } i_{k}=1, \ldots, N_{k}, k=1, \ldots, M .
\end{aligned}
$$

The decision problem of record linkage in the context of multipartite entity resolution is concerned with choosing a feasible partition $\gamma \in \Gamma$ that maximizes the posterior probability $P\left(\gamma \mid D^{M}\right)$ given the data, i.e.,

$$
\max _{\gamma \in \Gamma} P\left(\gamma \mid D^{M}\right) \text {. }
$$

Note that because multiplying the objective by the constant (e.g., $\left[P\left(\gamma^{0} \mid D^{M}\right)\right]^{-1} \equiv$ const for specific $\gamma^{0}$ ) does not change the solution of the optimization problem, (17) is equivalent to

$$
\max _{\gamma \in \Gamma} \frac{P\left(\gamma \mid D^{M}\right)}{P\left(\gamma^{0} \mid D^{M}\right)},
$$

where $\gamma_{0}$ denote a partition that consists of all missing records. In terms of $D_{i_{1} \ldots i_{M}}$, the partition $\gamma^{0}$ can be written as

$$
\gamma^{0}=\left\{D_{0 \ldots 0 i_{k} 0 \ldots 0} \mid i_{k}=1, \ldots, N_{k}, k=1, \ldots, M\right\} .
$$

Using Bayes' theorem:

$$
P(A \mid B)=\frac{P(B \mid A) P(A)}{P(B)}, \forall \text { events } A, B: P(B) \neq 0,
$$

we can write the posterior in $(17)$ and $(18)$ as:

$$
P\left(\gamma \mid D^{M}\right)=\frac{g\left(D^{M} \mid \gamma\right) P(\gamma)}{g\left(D^{M}\right)}
$$

where $g\left(D^{M}\right)$ and $g\left(D^{M} \mid \gamma\right)$ denote the joint probability density function of the observed data $D^{M}$ in $M$ databases and the conditional probability density function of the observed data $D^{M}$ given a partition $\gamma$, respectively. Note that partitions have a discrete distribution, while the observed data are supposed (for simplicity) to be continuous (although the latter could be written as mixture of discrete and continuous variables if needed).

It is reasonable to assume that given a partition individual record linkages are mutually independent. Hence, the likelihood can be written as:

$$
g\left(D^{M} \mid \gamma\right)=\prod_{D_{i_{1} \ldots i_{M}} \in \gamma} g\left(D_{i_{1} \ldots i_{M}} \mid \gamma\right),
$$


whereas the prior can be written as:

$$
\begin{aligned}
P(\gamma) & =\left(\int g\left(D^{M}\right) d D^{M}\right) \times \\
& \times \prod_{D_{i_{1} \ldots i_{M}} \in \gamma} \int g\left(D_{i 1 \ldots i_{M}} \mid \gamma\right) d D_{i 1 \ldots i_{M}} .
\end{aligned}
$$

Using (22) and (23), the posterior 21) can be rewritten as

$$
\begin{aligned}
& P\left(\gamma \mid D^{M}\right)=\frac{\int g\left(D^{M}\right) d D^{M}}{g\left(D^{M}\right)} \times \\
& \times \prod_{D_{i_{1} \ldots i_{M}} \in \gamma} g\left(D_{i_{1} \ldots i_{M}} \mid \gamma\right) \cdot\left(\int g\left(D_{i 1 \ldots i_{M}} \mid \gamma\right) d D_{i 1 \ldots i_{M}}\right)
\end{aligned}
$$

Additionally, if $\gamma, \rho \in \Gamma$ are two distinct partitions (i.e., $\gamma \neq \rho$ ) and $D_{i_{1} \ldots i_{M}} \in$ $\gamma \cap \rho$, we assume that the following holds:

$$
\begin{aligned}
& g\left(D_{i_{1} \ldots i_{M}} \mid \gamma\right) \cdot\left(\int g\left(D_{i 1 \ldots i_{M}} \mid \gamma\right) d D_{i 1 \ldots i_{M}}\right)= \\
& =g\left(D_{i_{1} \ldots i_{M}} \mid \rho\right) \cdot\left(\int g\left(D_{i 1 \ldots i_{M}} \mid \rho\right) d D_{i 1 \ldots i_{M}}\right) .
\end{aligned}
$$

We can write the objective function in 18 as follows, using (24):

$$
\begin{aligned}
& \frac{P\left(\gamma \mid D^{M}\right)}{P\left(\gamma^{0} \mid D^{M}\right)}= \\
= & \prod_{D_{i_{1} \ldots i_{M}} \in \gamma} \frac{\left(D_{i_{1} \ldots i_{M}} \mid \gamma\right)}{J_{0}} \times\left(\int g\left(D_{i 1 \ldots i_{M}} \mid \gamma\right) d D_{i 1 \ldots i_{M}}\right),
\end{aligned}
$$

where

$$
J_{0}=\prod_{k=1, i_{k} \neq 0}^{M} g\left(D_{0 \ldots i_{k} \ldots 0} \mid \gamma^{0}\right)\left(\int g\left(D_{0 \ldots i_{k} \ldots 0} \mid \gamma^{0}\right) d D_{0 \ldots i_{k} \ldots 0}\right)
$$

Applying a negative natural logarithm to both sides (26), we have:

$$
\begin{aligned}
& -\ln \left(\frac{P\left(\gamma \mid D^{M}\right)}{P\left(\gamma^{0} \mid D^{M}\right)}\right)= \\
& =\sum_{D_{i_{1} \ldots i_{M}} \in \gamma}\left\{\ln J_{0}-\ln g\left(D_{i_{1} \ldots i_{M}} \mid \gamma\right)\right. \\
& \left.-\ln \left(\int g\left(D_{i 1 \ldots i_{M}} \mid \gamma\right) d D_{i 1 \ldots i_{M}}\right)\right\} .
\end{aligned}
$$

Setting

$$
\begin{aligned}
c_{i_{1} \ldots i_{M}} & =\ln J_{0}-\ln g\left(D_{i_{1} \ldots i_{M}} \mid \gamma\right) \\
& -\ln \int g\left(D_{i 1 \ldots i_{M}} \mid \gamma\right) d D_{i 1 \ldots i_{M}}
\end{aligned}
$$


we obtain from 28 :

$$
-\ln \left(\frac{P\left(\gamma \mid D^{M}\right)}{P\left(\gamma^{0} \mid D^{M}\right)}\right)=\sum_{D_{i_{1} \ldots i_{M}} \in \gamma} c_{i_{1} \ldots i_{M}}
$$

and the maximum likelihood (18) with the constraints $(16)$ on partitions $\gamma \in \Gamma$ is restated as the following minimization problem, known as the multidimensional assignment or the MAP:

$$
\begin{aligned}
& \text { minimize } y:=\sum_{i_{1}=0}^{N_{1}} \cdots \sum_{i_{M}=0}^{N_{M}} c_{i_{1} \ldots i_{M}} x_{i_{1} \ldots i_{M}} \\
& \text { subject to } \\
& \qquad \sum_{\left(N_{1}, \ldots, N_{k-1}, N_{k+1}, \ldots, N_{M}\right)} x_{i_{1} \ldots i_{M}}=1 \\
& \left(i_{1}, \ldots, i_{k-1}, i_{k+1}, \ldots, i_{M}\right)=(0, \ldots, 0) \\
& \text { for } i_{k}=0,1, \ldots, N_{k}, k=1, \ldots, M .
\end{aligned}
$$

We assume that the cost coefficients in 29 in the optimization problem (31) are proportional to the corresponding $\delta$ in (2). This assumption is natural for the setting of user profile matching. In fact, any cost coefficient $c_{i_{1} \ldots i_{M}}$ in 29 corresponds to negative log-likelihood of the matching of records $\left(i_{1}, \ldots, i_{M}\right)$, where the records are ordered in accordance with datasets. For $c_{i_{1} \ldots i_{M}}$, we have $\delta\left(i_{1}, \ldots, i_{M}\right)=\sum_{k=1}^{M} \sum_{j<k}\left(1-\operatorname{sim}\left(i_{j}, i_{k}\right)\right)=(M-1) M / 2-$ $m \operatorname{sim}\left(i_{1}, \ldots, i_{M}\right)$, and so $\exp \left\{-\delta\left(i_{1}, \ldots, i_{M}\right)\right\} \propto m \operatorname{sim}\left(i_{1}, \ldots, i_{M}\right)$. Hence, $\exp \left\{-c_{i_{1} \ldots i_{M}}\right\} \propto m \operatorname{sim}\left(i_{1}, \ldots, i_{M}\right)$. In other words, the corresponding likelihood of matched records $\left(i_{1}, \ldots, i_{M}\right)$ is proportional to the multi-record similarity $m \operatorname{sim}\left(i_{1}, \ldots, i_{M}\right)$. Of course, the minimization of negative log-likelihood is simply a maximum likelihood approach. Naturally, in user profile matching, more similar records are more likely to represent a better match.

\section{Solution of the multidimensional assignment problem (MAP)}

The solution approaches for the MAP include a number of exact and approximate methods ranging from branch-and-bound [39] and Lagrangian relaxation [13] to tri-substitution [38] and such metaheuristics as tabu search, simulated annealing, greedy randomized adaptive search procedure, memetic approach, and hybridization of several techniques [27. The problem received an increased attention in the last decade [19, 25, 29, 30, 33, 35, 37, 49, Here we will utilize and discuss two solution algorithms, namely a Greedy algorithm 33. and very large-scale neighborhood search [26]. 
4.1 Greedy algorithm for the MAP with discrete costs

Here we describe a greedy solution algorithm proposed in 33 for solving the MAP with discrete costs. This algorithm works by iteratively finding the smallest cost coefficient $c_{i_{1} \ldots i_{M}}$ and then removing cost coefficients $c_{j_{1} \ldots j_{M}}$ with $i_{k}=j_{k}$ for any $k=1, \ldots, M$ (so that infeasible solutions cannot be selected). With an exception of the notation, here we will follow Algorithm 1 in [33], including the assumption of equal cardinalities $N_{1}=\ldots=N_{M}=n$ for all dimensions. Further research is needed to adapt this algorithm to work for a general case with different cardinalities, possibly by introducing dummy records in some dimensions to associate with partial profile matches.

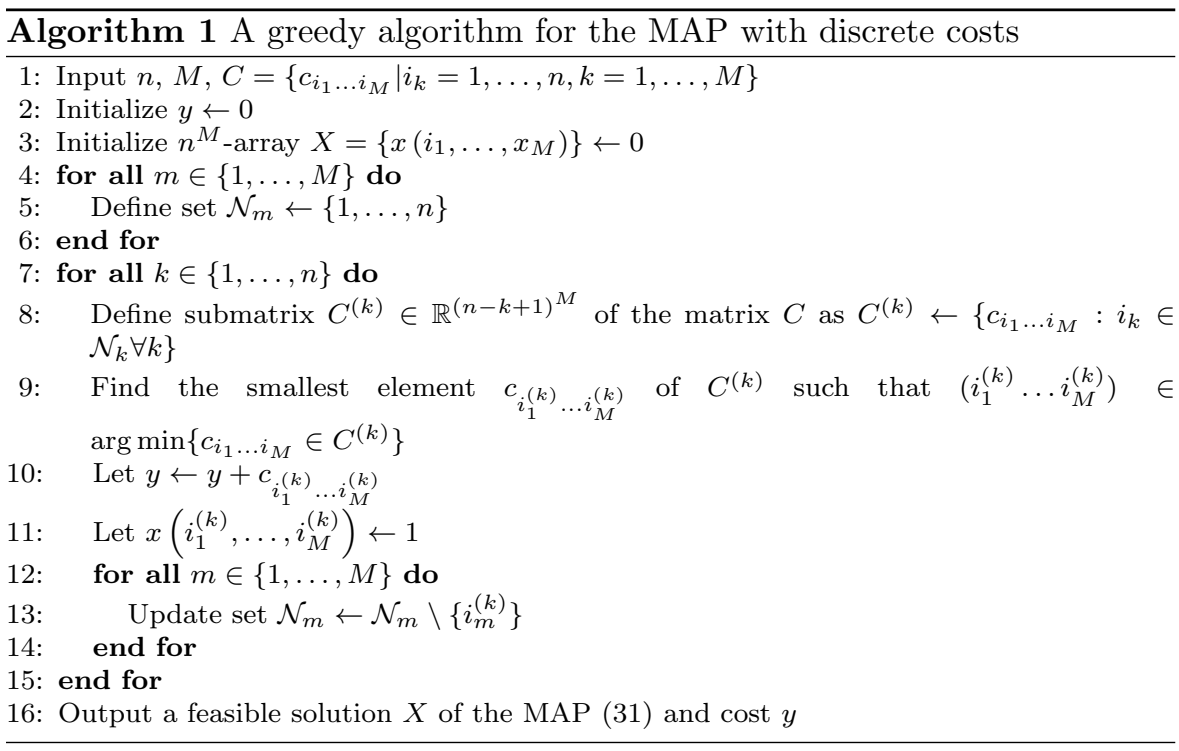

\subsection{Very large-scale neighborhood (VLSN) local search}

We describe the solution algorithm that was recently proposed in 26]. Specifically, we present the version of the VLSN search algorithm for the MAP that is based on finding an optimal permutation for each dimension. The search alternates between two phases: (i) the exploitation phase (or a descend to a local minimum) and (ii) the exploration phase (or a re-initialization of a descent from a different solution).

We will follow the notation introduced in 26 . For convenience, we assume $N_{1}=\ldots=N_{M}=n$, i.e., cardinalities are the same in each dimension. The algorithm can be modified to allow for different cardinalities. For example, one can always index the datasets in a decreasing order of contained records and introduce enough dummy records into datasets with smaller cardinalities. 
We take advantage of a well-known alternative formulation of the MAP 31 using the combinatorial optimization representation of the MAP solutions as the ordered set of $M-1$ permutations of size $n$, where $M, n$ are dimensionality and cardinality of the problem (see, e.g., [7). Let $S$ denote any given feasible solution of the MAP. Solution $S$ could be some starting solution or the current solution derived by changing some starting solution to preserve feasibility (i.e., ensure that all the constraints remain satisfied). For instance, a starting solution can be constructed as a feasible solution represented combinatorially by $M-1$ identity permutations $(1,2, \ldots, n)^{T}$. Another example of a starting solution is an ordered set of $M-1$ randomly generated permutations).

Let $k$ be dimension and $S=\left(\pi_{2}, \ldots, \pi_{M}\right)$ be a solution of an instance of the MAP (31) with cost coefficients $C$. An $n \times n$ matrix $C(k, S)$ with elements $C_{(k, S)}[i, j]$ is a projection of the solution $S$ onto the multidimensional matrix $C$ without (with free) dimension $k$ is defined as

$$
\begin{array}{r}
C_{(k, S)}[i, j]=C\left[i, \pi_{2}(i), \ldots, \pi_{k-1}(i), j, \pi_{k+1}(i), \ldots, \pi_{M}(i)\right], \\
C_{(1, S)}[i, j]=C\left[i, \pi_{2}(j), \ldots, \pi_{M}(j)\right], \\
\text { for } i=1, \ldots, n ; j=1, \ldots, n .
\end{array}
$$

Consider the linear assignment problem (LAP) with an $n \times n$ matrix of cost coefficients $B=\left(b_{i j}\right)_{i=1, \ldots, n ; j=1, \ldots, n}$ :

$$
\min _{\pi \in \Pi_{n}} \sum_{i=1}^{n} b_{i \pi(i)} .
$$

Let $\operatorname{LAP}(B)=(\sigma, y(\sigma))$ denote a solution $\sigma \in \Pi_{n}$ with objective value $y=$ $y(\sigma)$ of the LAP $(34)$ with coefficient matrix $B$.

This algorithm works by using a multi-start for exploration and solving the respective two-dimensional assignments for exploitation of a very large neighborhood. First by finding a projection of the multidimensional costs in dimension $k$, the algorithm computes the $n \times n$ cost matrix $C(k, S)$ to be used in solving the linear assignment problem (LAP). Then the solution of the LAP replaces the $k$-th permutation-column in the current solution, and the algorithm continues in the next dimension $k+1$ for $k<M$ or in the dimension 1 for $k=M$, until no improvement is possible.

Example of input problem instance for algorithm 2 is depicted at figure 1. solution for this problem instance is depicted at figure 2. Nodes of the graph at figure 2 contain objective function value, directed edges are constructed by solving LAP.

Figure 2 shows example output graph generated by algorithm 2 with input matrix as in figure 1.

In Algorithm 2 the steepest descent through dimensions is described. Besides the steepest descent, the following variants of this search can be considered.

- Best improvement-based solution update (place a taboo on checking dimension $k^{\prime}$ in the next step). 


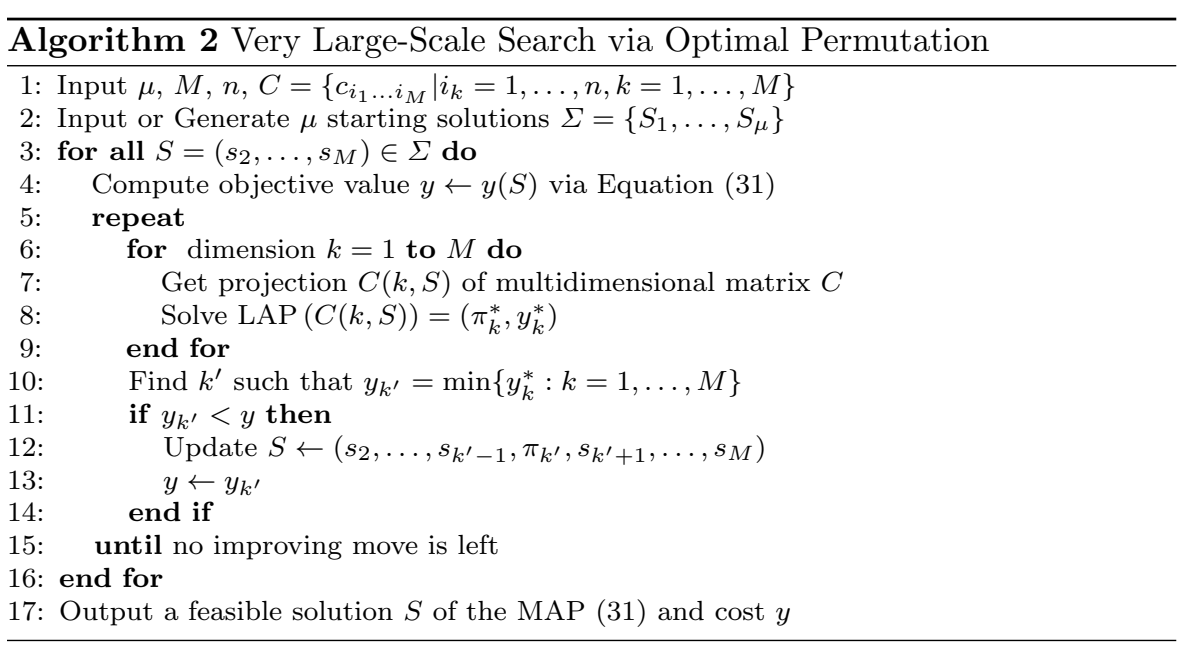

\begin{tabular}{|r|r|r|}
\hline 693 & 933 & 203 \\
\hline 931 & 106 & 126 \\
\hline 376 & 712 & 343 \\
\hline & & \\
\hline 800 & 564 & 934 \\
\hline 944 & 542 & 150 \\
\hline 300 & 442 & 671 \\
\hline & & \\
\hline 756 & 820 & 579 \\
\hline 91 & 596 & 76 \\
\hline 110 & 771 & 997 \\
\hline
\end{tabular}

Fig. 1 Three dimensional input hypermatrix example for algorithm 2

- First improvement-based solution update (continue in new directions by increasing dimensions $k=k^{\prime}+1, \ldots$ or revisit previous directions by decreasing $k=k^{\prime}-1, \ldots$, placing a taboo on checking dimension $k^{\prime}$ in the next step).

- Random choice among improving solutions (with taboo on dimension $k^{\prime}$ in the next iteration).

4.3 Multi-start strategies for the VLSN search

The exploration stage of the VLSNS metaheuristic includes construction of another feasible solution from which the local search is to be restated. Multi-start strategy is a systematic way of constructing starting solutions. The original paper [26] that introduces the VLSNS metaheuristic describes two types of multi-start strategies, namely a random sampling strategy and a design based strategy. Using design based multi-start strategy in [26], we propose a grid based strategy and compare the grid-based multi-start to random sampling multi-start. In particular, we modify the (random) design based restarts into 


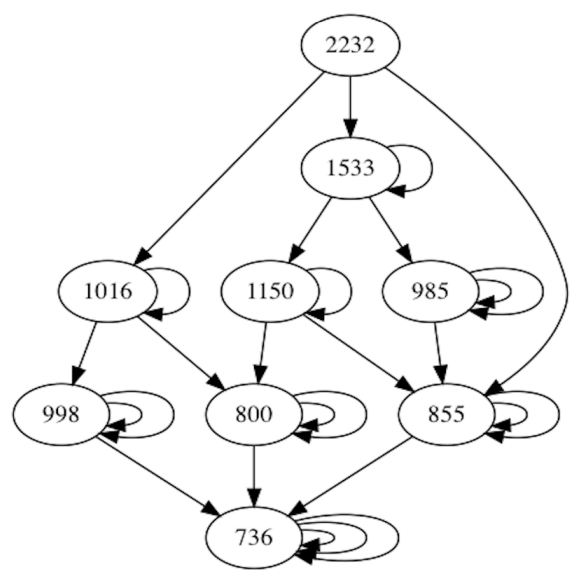

Fig. 2 VLSN for matrix at figure 1 Starting solution is $(693,542,997)=2232$; result is $(203,442,91)=736$

the grid based restarts by using an identity permutation in place of a randomly generated permutation in the construction of starting solutions.

In combinatorics, a cyclic permutation is typically defined as a permutation which shifts all its elements $\{1, \ldots, N\}$ by a fixed offset, so that the elements moved off the end are inserted at the beginning of the permutation. In [26], authors consider two types of cyclical permutations applied on a given permutation $\pi=(\pi(1), \ldots, \pi(N))$ of $N$-element set, namely

(i) a $k$-push-up $\nu_{k}$ :

$$
\nu_{k}(\pi(i))=\left\{\begin{aligned}
\pi(i+k) & \text { for } i=1, \ldots, N-k, \\
\pi(i+k-N) \text { for } i & =N-k+1, \ldots, N .
\end{aligned}\right.
$$

(ii) a $k$-push-down $\delta_{k}$ :

$$
\delta_{k}(\pi(i))=\left\{\begin{aligned}
\pi(N+i-k) \text { for } i & =1, \ldots, k \\
\pi(i-k) \text { for } i & =k+1, \ldots, N .
\end{aligned}\right.
$$

where $k$ is an integer $k=1, \ldots, N-1$.

\section{Complexity}

The MAP is NP-hard (Karp, 1972). Next, we discuss an approach to evaluating the complexity of the VLSN search. In particular, we show that the search tree admits a partition of its nodes into the levels. These levels are ordered based on the MAP dimensions, which can be searched further. We use the partition of nodes into the levels in the search tree to define two related absorbing Markov chains. These two Markov chains allow us to obtain the upper bound on the expected number of moves through partition levels until reaching a local optimum. 


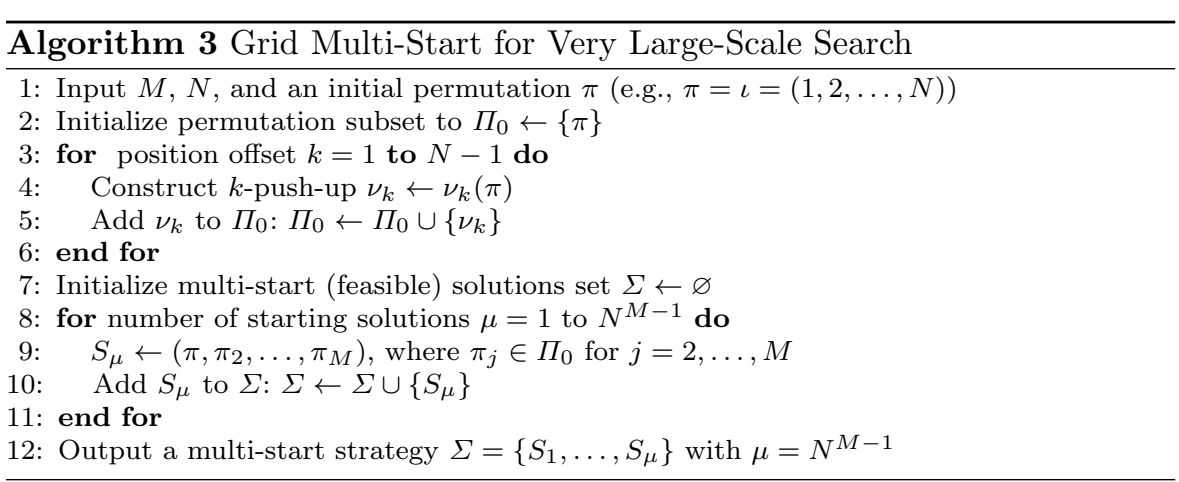

To evaluate complexity of the VLSN search algorithm, observe that this algorithm works by successively searching through dimensions $1,2, \ldots, M$. Let $y$ and $y^{\prime}$ denote objective values of the current solution $S$ and the neighboring solution $S^{\prime}$. A starting solution or the current solution $S$ has some $I \leq M$ dimensions $m_{1}, m_{2}, \ldots, m_{I}$ that lead to improved objective value $y^{\prime}<y$. For the remaining $M-I \geq 0$ dimensions, there is no improvement as the neighborhood search returns $y^{\prime}=y$. Let us refer to the former $I$ dimensions as the improving dimensions of the current solution $S$, while the latter $M-I$ dimensions are called non-improving dimensions. The numbers of improving and non-improving dimensions $I, M-I \in\{0,1, \ldots, M\}$. This introduces a natural partition of the search tree of this algorithm into $M+1$ levels according to the specific number $I$ of improving dimensions that remain.

To illustrate this partition, consider, for example, a search tree in Fig. 2 for the MAP with $M=3, n=3$. The source node depicts a starting solution with objective value 2232 . The source node has an outdegree of three that corresponds to three improving dimensions, i.e., the source node belongs to the $I=3$ level. The intermediate nodes with objective values 1533, 1150, 1016 have outdegree two each (not counting the self-loops) and belong to the $I=2$ level. Whereas the intermediate nodes with objective values 998, 985, 855, 800 have outdegree one each and belong to the $I=1$ level. The sink node with the (local and global) minimum objective value 736 has a zero outdegree and belongs to the $I=0$ level in the search tree. Observe that the $I=2$ level's node with value 1533 leads to either another node (value 1150) on the same $I=2$ level or the node (value 985$)$ on the lower level $I=1(=2-1)$. Interestingly, the source node (value 2232) cannot stay on the same top level $I=3$ and instead leads to either the $I=2$ level nodes or the $I=1$ level node (value 855). That is because an improving dimension that is used cannot be replaced with any other previously improved-on dimension.

As the algorithm progresses, the current solution moves from $S$ with $y$ and $I$ to its neighboring solution $S^{\prime}$ with $y^{\prime}<y$ and $I^{\prime}$. It is easy to see that for the VLSN search algorithm, we have:

(a) if $I=M$ then $I^{\prime} \in\{I-1, I-2, \ldots, 0\}$;

(b) if $I=1, \ldots, M-1$ then $I^{\prime} \in\{I, I-1, \ldots, 0\}$; 
(c) if $I=0$ then $I^{\prime}=\emptyset$.

In fact, $S$ and $S^{\prime}$ only differ in one dimension $k^{*}$, and by construction of the VLSN search no further improvements can be made through that dimension from $S^{\prime}$. If the current solution $S$ has $I=M$ then this solution is on the top level of the search tree. So, there are no dimensions that have been explored before such $S$. That means that its neighborhood solution $S^{\prime}$ cannot remain on the top level. Instead such $S^{\prime}$ must have $I^{\prime}<I$. On the other hand, if the current solution $S$ has $0<I<M$ then its neighborhood solution $S^{\prime}$ could have an improvement in some previously explored dimension $k_{1}$ (through which $S^{\prime}$ would have a neighbor $S_{1}$ with value $y_{1}$ ) as long as $y_{1}<y^{\prime}$. This means that for the levels $I=1, \ldots, M-1$, it is possible for $S^{\prime}$ to remain at the same level $I$ as $S$ after an objective-value improving move. Obviously, if the current solution $S$ has $I=0$ then no dimensions with improving moves remain, and so the current solution is a local (or global) optimum.

Next, we define two related Markov chains that allow us to evaluate the expected number of transitions through intermediate levels before reaching a minimum. The first Markov chain corresponds with the VLSN search through the levels, with the $I=0$ level as an absorbing state. The second Markov chain simplifies the evaluation of the first passage times in comparison with the first Markov chain.

For any $I_{0}, I_{1}=0,1, \ldots, M$, let $p\left(I_{0}, I_{1}\right)=\operatorname{Pr}\left(I_{0}^{\prime}=I_{1}\right)$ denote the probability of the number of improving dimensions changing from $I_{0}$ to $I_{1}$ when the VLSN search moves from $S_{0}$ and $S_{1}$. Now let us define a Markov chain $C$ with $M+1$ states corresponding to the levels of the search tree with a given number $I$ of the remaining improving dimensions. This Markov chain has an absorbing state at $I=0$, as the VLSN search algorithm stops when a local optimum is reached and no improving moves can be made. Consider the transition matrix $P=\left(P_{i j}\right)_{i, j=0}^{M}$ of this Markov chain $C$.

$$
P=\left(\begin{array}{cccccc}
1 & 0 & 0 & \ldots & 0 & 0 \\
p(1,0) & p(1,1) & 0 & \ldots & 0 & 0 \\
\vdots & \vdots & \vdots & \ddots & \vdots & \vdots \\
p(M-1,0) & p(M-1,1) & & \ldots & p(M-1, M-1) & 0 \\
p(M, 0) & p(M, 1) & p(M, 2) & \ldots & p(M, M-1) & 0
\end{array}\right)
$$

To get an upper bounds for the expected number of transitions before reaching the absorbing state for Markov chain $C$, consider a related Markov chain $C_{1}$ whose transition matrix $P_{1}$ is based on the transition probabilities.

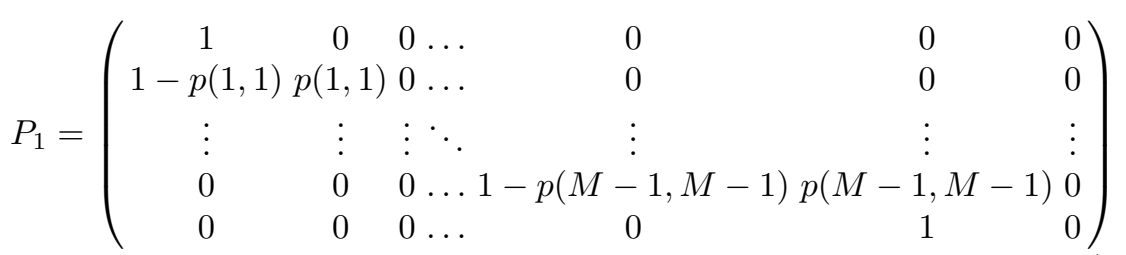


Let $\nu_{I}, I=0,1, \ldots, M$ denote the expected number of transitions in this Markov chain $C_{1}$ starting in state $I$ before reaching the absorbing state $I=0$, i.e. local minimum. Note that $\nu_{0}=0$ and for any $0<I<M, \nu_{I}=1+$ $p_{I, I} \nu_{I}+\left(1-p_{I, I}\right) \nu_{I-1}$ or $\nu_{I}=\nu_{I-1}+\frac{1}{1-p_{I, I}}$. Hence, $\nu_{I}=\sum_{i=1}^{I} \frac{1}{1-p_{i, i}}$ for $I=1,2, \ldots, M-1$. Also, $\nu_{M}=1+\nu_{M-1}=1+\sum_{i=1}^{M-1} \frac{1}{1-p_{i, i}}$.

The Hungarian algorithm for solving the LAP in each dimension has complexity of order $n^{3}$. Whereas the complexity of extracting the matrix of cost coefficients for the LAP (i.e., getting the projection $C(k, S)$ of multidimensional cost array $C$ ) has order $n^{2}$. The expected number of times the VLSN search repeatedly solves the Hungarian algorithm does not exceed $\mu M \nu_{M}=$ $\mu M\left(1+\sum_{i=1}^{M-1} \frac{1}{1-p_{i, i}}\right)$. Hence, the VLSN search has the worst-case complexity of $n^{3} \mu M \nu_{M}$.

\section{Numerical experiments}

The main goal of our numerical studies is to document and compare the performance of two recent heuristics developed for general MAPs of large size. Additionally, we want to understand the extent to which different exploration or multi-start strategies can influence the solutions returned by the VLSN search algorithm. This is particularly important because [26] proposed two distinct multi-start strategies but have not studied or compared them numerically.

Specifically, we have implemented two algorithms described above, and performed experiments, aimed at answering the following questions:

1. Does the VLSN search result in better MAP solutions than those produced by Greedy algorithm?

2. Does a multi-start VLSN search strategy improve the found solution when compared to a single-start?

3. Does the use of greedy algorithm's solution as the starting solution for the VLSN search improve the found solution when compared to the VLSN search started at a randomly constructed feasible solution?

4. How does the grid compare to the random start?

The algorithms were implemented in $\mathrm{C}++$; the code was executed in sequential manner using 40 core Intel(R) Xeon(R) CPU E5-2640 v4 @ 2.40GHz processor with 256 GB RAM. Optimal solutions were obtained using Gurobi 9.0 solver on the same computer, using 20 threads. In Gurobi, we have set timelimit 10 minutes for each of the problems. While running VLSN we have set limit on number of explored nodes for instances with $\mathrm{M}=3$ and $\mathrm{N}=$ 50 (250000 nodes), and $\mathrm{N}=100$ (400000 nodes), and with $\mathrm{M}=4, \mathrm{~N}=20$ (1600000 nodes), and $\mathrm{N}=30$ (2700000 nodes).

We have implemented two versions of genetic algorithm, inspired by a random-key generic algorithm as described in [48. As MAP is a constrained discrete optimization problem, we had to develop an encoding and decoding 
scheme that would satisfy problem constraints after the crossover operations. We have represented a chromosome as a matrix $M_{c d}$ where number of rows $c$ equals to problem cardinality, and number of columns $d$ to dimensionality of considered MAP. Then, each row of the matrix would represent coordinates of the cell of the MAP hypermatrix. Each column $i \in(1, \ldots d)$ of $M_{c d}$ represents a permutation of elements $(1, \ldots, c)$. MAP objective function value would then be equal to the sum of values corresponding to all cells in $M_{c d}$. Crossover operation producing one chromosome from two was implemented as a random combination of columns of two matrices $M_{c d}^{1}$ and $M_{c d}^{2}$. Each evolution step was taking $p \%$ of the best ("elitist") solutions, performing crossover resulting in $r \%$ of new chromosomes, merging the results with $q<p \%$ top solutions and generating remaining population randomly. We have implemented two versions of genetic algorithm: GA1 had $p$ set to $60 \%, q$ to $20 \%$, and $r$ to $60 \%$, and GA2 had $p$ set to $40 \%$ (thus, only "elite" $20 \%$ and remaining $20 \%$ were involved in the crossover). GA1 had 2000 evolution steps, GA2 had 5000 steps.

We have generated random hypermatrices of different dimensions $M$ and cardinalities $N$, and solved the MAP for these synthetic matrices. Matrix elements contain uniformly distributed random numbers in the range from 0 to 1 . Results of the experiment are shown in Tables 2 and 3 . These tables show pairwise comparisons of several versions of the VLSN search with greedy algorithms, genetic algorithms, and all-purpose solver Gurobi 9. These include the VLSN search with a single starting solution (denoted by VLSN1), the VLSN search with multiple starts (denoted by VLSNMS), a greedy algorithm (denoted by Greedy), the VLSN search which uses deterministic grid-based strategy to construct starting feasible solutions (denoted by GridVLSN), and a hybridization of the VLSN search with greedy algorithm, where the VLSN search uses the solution found by greedy procedure as a starting solution. For each of these algorithms, we record the (best or smallest local optimal) values $y_{\text {alg }}^{i}$ of the solutions found by the algorithm for a given problem instance $i \in\{1, \ldots, I\}$ and compare these values using statistical approaches.

We present and discuss the results of statistical comparison of VLSN and non-VLSN algorithms in Section 6.1. As the size $N^{M}$ of hypermatrix of cost coefficients grows exponentially with the dimensionality $M$, we limit our numerical experiments to the MAP instances with $M=3,4$. For the 3 -AP, we consider instances with cardinality $N=3,10,20,30,40,50,100$. While for the 4 -AP, we consider $N=3,10,20,30$. Although the MAP instances with $N=3$ are trivial having the input size of 27 or 81 cost coefficients, the larger cardinality $N$ values in our experiments produce hypermatrices with $1,000,000$ and 810,000 coefficients and instances with $100 !^{2}$ and $30 !^{3}$ feasible solutions for this generally NP-hard problem.

Furthermore, we illustrate the application of our MAP-based approach to solving multipartite entity resolution problem from computer science in Section 6.2. We show that VLSN-based approaches such as Greedy-VLSN can perform well when matching data from three datasets or sources with various degree of dissimilarity in the records contained in different sources (i.e., datasets). 
6.1 Statistical comparison of the solution quality for the VLSN search and alternative algorithms

We compare the quality of solutions found by various versions of the VLSN search and alternative algorithms, including metaheuristics, Greedy heuristic, and a standard solver such as Gurobi. In particular, the comparisons are performed on sets of randomly generated problem instances for given cardinality $(n)$ and dimensionality $(M)$ parameters of the MAP. To perform proper statistical comparisons among the algorithms, we fix the MAP parameters $n, M$ and choose pairs $\left(A_{1}, A_{2}\right)$ of algorithms to compare so that Algorithm $1, A_{1}$, is a version of the VLSN search (e.g., a grid multi-start VLSN search, or a hybrid VLSN search with a start at Greedy solution), and Algorithm 2, $A_{2}$ is an alternative, non-VLSN algorithm (e.g., a Genetic algorithm GA, a Greedy algorithm, Gurobi, etc.).

Let $y_{1}, y_{2}$ (and $T_{1}, T_{2}$ ) denote the objective values (and the number of solutions explored) that are found by algorithms $A_{1}, A_{2}$. Recall that $A_{1}$ always stands for some VLSN algorithm while $A_{2}$ is non-VLSN, and consider a difference $y_{1}-y_{2}$ between their returned objective values (alternatively, $T_{1}-T_{2}$ is a difference in the number of searched solutions between $A_{1}$ and $A_{2}$ ). This difference $y_{1}-y_{2}$ is positive, when the quality of solution found by the VLSN search is better than its alternative's $A_{2}$. For some of $I=100$ randomly generated problem instances, the $y_{1}-y_{2}$ differences are randomly distributed. Recall that the objective values $y_{1}, y_{2}$ are sums of randomly generated cost coefficients. So, by the law of large numbers, for large cardinality $n$, the difference $y_{1}-y_{2}$ is approximately normally distributed as a difference of approximately normal $y_{i}, i=1,2$.

To statistically compare the solution qualities, we construct a confidence interval for $y_{1}-y_{2}$, where the observed differences are taken between $y_{1}=y_{1}(i)$ and $y_{2}=y_{2}(i)$ when $A_{1}, A_{2}$ are ran on the same problem instance $\operatorname{MAP}_{i}(n, M)$ where $n, M$ are the cardinality and the dimensionality, and $i=1,2, \ldots, I$. To construct the confidence intervals, find the sample mean

$$
A_{1,2}=\widehat{y_{2}-y_{1}}=\frac{1}{I} \sum_{i=1}^{I}\left(y_{2}(i)-y_{1}(i)\right) \text {, }
$$

and the sample standard deviation $D_{1,2}=\sqrt{\frac{1}{I-1} \sum_{i=1}^{I}\left(y_{2}(i)-y_{1}(i)-A_{1,2}\right)^{2}}$. Given a p-value of 0.05 , the $95 \%$-confidence interval for the difference $y_{2}-y_{1}$ is

$$
\left(A_{1,2}-t_{1-\alpha / 2, I-1} \cdot \frac{D_{1,2}}{\sqrt{I-1}}, \quad A_{1,2}+t_{1-\alpha / 2, I-1} \cdot \frac{D_{1,2}}{\sqrt{I-1}}\right),
$$

where $t_{\alpha}$ is the $\alpha$-th percentile of the t-distribution. Here, we use $\alpha=0.05$, $I-1=99$, and $t_{1-\alpha / 2, I-1}=t_{0.975,99}=1.984$.

We compute $A_{1,2}, D_{1,2}$, and the confidence intervals for all possible combinations of the VLSN search variants $A_{1}$ and the alternative, non-VLSN 
solution algorithms $A_{2}$. Using the confidence intervals, we interpret the results of comparison as follows. If zero does not belong to the confidence interval, then the difference in performance (i.e., solution quality) between $A_{1}$ and $A_{2}$ is statistically significant. Moreover, the VLSN search outperforms if $0<A_{1,2}-t_{1-\alpha / 2, I-1} \cdot \frac{D_{1,2}}{\sqrt{I-1}}$, or equivalently $A_{1,2}>t_{1-\alpha / 2, I-1} \cdot \frac{D_{1,2}}{\sqrt{I-1}}$. Otherwise the non-VLSN algorithm outperforms if $A_{1,2}+t_{1-\alpha / 2, I-1} \cdot \frac{D_{1,2}}{\sqrt{I-1}}<0$, or $A_{1,2}<-t_{1-\alpha / 2, I-1} \cdot \frac{D_{1,2}}{\sqrt{I-1}}$.

Observe that most pairs of VLSN and non-VLSN algorithms $A_{1}, A_{2}$ are not based on each other. The only exception from this rule is comparison between Greedy-VLSN (a hybridized VLSN with Greedy solutions as its starts) and Greedy (non-VLSN). By construction, Greedy-VLSN is guaranteed to always do at least as good as Greedy on the same problem instance. So, the differences of these two will follow a log-normal distribution rather than be approximately normally distributed, since there is a dependence. Hence, we modify the construction of the confidence intervals for these two algorithms.

First, for the Greedy-VLSN and Greedy algorithms $A_{1}, A_{2}$, we calculate the sample means and standard deviations for the logged differences:

$$
\left.A_{1,2}=\log \widehat{\left(y_{2}-\right.} y_{1}\right)=\frac{1}{I} \sum_{i=1}^{I} \log \left(y_{2}(i)-y_{1}(i)\right),
$$

and

$$
D_{1,2}=\sqrt{\frac{1}{I-1} \sum_{i=1}^{I}\left(\log \left(y_{2}(i)-y_{1}(i)\right)-A_{1,2}\right)^{2}},
$$

where $\log (\cdot)$ represents a natural logarithm.

Using a modified Cox method [55] suggested for moderate sample sizes $I=100 \geq 50$, a $95 \%$ confidence interval for the log-difference $\log \left(y_{2}-y_{1}\right)$ between Greedy-VLSN $A_{1}$ and Greedy $A_{2}$ is

$$
(\log L, \log U)=\left(A_{1,2}+\frac{D_{1,2}^{2}}{2} \pm t_{1-\alpha / 2} \sqrt{\frac{D_{1,2}^{2}}{2}+\frac{D_{1,2}^{4}}{2(I-1)}}\right),
$$

where $t_{\alpha}$ is the $\alpha$-th percentile of the t-distribution. Here again, we use $\alpha=$ $0.05, I-1=99$, and $t_{1-\alpha / 2, I-1}=t_{0.975,99}=1.984$. Then a $95 \%$ confidence interval for the difference $\left(y_{2}-y_{1}\right)$ between Greedy-VLSN $A_{1}$ and Greedy $A_{2}$ is

$$
(L, U)=\left(\exp \left\{A_{1,2}+\frac{D_{1,2}^{2}}{2} \pm t_{1-\alpha / 2} \sqrt{\frac{D_{1,2}^{2}}{2}+\frac{D_{1,2}^{4}}{2(I-1)}}\right\}\right),
$$

The results of the comparison for the MAP with $M=3$ and $M=4$ are summarized in Tables 2 and 3 respectively. With exception of the small-size MAP with $M=3, n=3$ or $M=4, n=4$, VLSN algorithms outperforms 
Greedy and both genetic algorithms. As the cardinality and the size of instances grow, the random multi-start search (VLSNMS) seems to perform the best among the tested heuristics, as evident from the comparison with Gurobi. However, the deterministic grid-based multi-start (Grid-VLSN) begin to outperform the random multi-start search as the cardinality becomes sufficiently high (e.g., the 3 -APs with $n=100$ and the 4-APs with $n=20,30$. This suggests that due to the space-filling property of this design-based multistart 26], the grid-based multi-start strategy tends to result in exploration of more diverse or expansive set of solutions. On the other hand, the use of Greedy solution as a start in the VLSN search may improve the performance. In fact, for the 3-APs with $N=50$ and $N=100$, hybrid Greedy-VLSN search significantly outperforms other tested heuristics.

Table 2: Statistical Comparison of Experiments for $M=3$

\begin{tabular}{|c|c|c|c|c|c|c|c|}
\hline $\begin{array}{l}A_{1}: \\
\text { VLSN }\end{array}$ & $\begin{array}{l}A_{2}: \\
\text { Non- } \\
\text { VLSN }\end{array}$ & M & $\mathbf{n}$ & $\begin{array}{l}\text { Mean } \\
A_{1,2}\end{array}$ & Std. $D_{1,2}$ & 95\% C.I. & Winner \\
\hline \multirow{4}{*}{ VLSN1 } & Greedy & 3 & 3 & 214342.030 & 229466.388 & $(168586.546,260097.514)$ & VLSN \\
\hline & GA1 & 3 & 3 & -7695.080 & 45128.527 & $(-16693.686,1303.526)$ & None \\
\hline & GA2 & 3 & 3 & -7695.080 & 45128.527 & $(-16693.686,1303.526)$ & None \\
\hline & Gurobi & 3 & 3 & -7695.080 & 45128.527 & $(-16693.686,1303.526)$ & None \\
\hline \multirow{4}{*}{ VLSNMS } & Greedy & 3 & 3 & 223494.530 & 223403.268 & $(178948.029,268041.031)$ & VLSN \\
\hline & GA1 & 3 & 3 & 1457.420 & 12369.498 & $(-1009.052,3923.892)$ & None \\
\hline & GA2 & 3 & 3 & 1457.420 & 12369.498 & $(-1009.052,3923.892)$ & None \\
\hline & Gurobi & 3 & 3 & 1457.420 & 12369.498 & $(-1009.052,3923.892)$ & None \\
\hline \multirow{4}{*}{$\begin{array}{l}\text { Grid- } \\
\text { VLSN }\end{array}$} & Greedy & 3 & 3 & 223494.530 & 223403.268 & $(178948.029,268041.031)$ & VLSN \\
\hline & GA1 & 3 & 3 & 1457.420 & 12369.498 & $(-1009.052,3923.892)$ & None \\
\hline & GA2 & 3 & 3 & 1457.420 & 12369.498 & $(-1009.052,3923.892)$ & None \\
\hline & Gurobi & 3 & 3 & 1457.420 & 12369.498 & $(-1009.052,3923.892)$ & None \\
\hline \multirow{4}{*}{$\begin{array}{l}\text { Greedy- } \\
\text { VLSN }\end{array}$} & Greedy & 3 & 3 & 209645.460 & 230090.725 & $(3225943.686,810173971774378.625)$ & VLSN \\
\hline & GA1 & 3 & 3 & -12391.650 & 41144.578 & $(-20595.858,-4187.442)$ & Non-VLSN \\
\hline & GA2 & 3 & 3 & -12391.650 & 41144.578 & $(-20595.858,-4187.442)$ & Non-VLSN \\
\hline & Gurobi & 3 & 3 & -12391.650 & 41144.578 & $(-20595.858,-4187.442)$ & Non-VLSN \\
\hline \multirow{4}{*}{ VLSN1 } & Greedy & 3 & 10 & 542017.770 & 315482.629 & $(479110.691,604924.849)$ & VLSN \\
\hline & GA1 & 3 & 10 & 760288.510 & 175283.909 & $(725336.986,795240.034)$ & VLSN \\
\hline & GA2 & 3 & 10 & 769004.430 & 181455.812 & $(732822.231,805186.629)$ & VLSN \\
\hline & Gurobi & 3 & 10 & -128186.000 & 70074.942 & $(-142158.909,-114213.091)$ & Non-VLSN \\
\hline \multirow{4}{*}{ VLSNMS } & Greedy & 3 & 10 & 665923.850 & 313330.992 & $(603445.806,728401.894)$ & VLSN \\
\hline & GA1 & 3 & 10 & 884194.590 & 154886.611 & $(853310.277,915078.903)$ & VLSN \\
\hline & GA2 & 3 & 10 & 892910.510 & 167886.376 & $(859434.050,926386.970)$ & VLSN \\
\hline & Gurobi & 3 & 10 & -4279.920 & 12186.237 & $(-6709.850,-1849.990)$ & Non-VLSN \\
\hline \multirow{3}{*}{$\begin{array}{l}\text { Grid- } \\
\text { VLSN }\end{array}$} & Greedy & 3 & 10 & 629492.110 & 318919.295 & $(565899.761,693084.459)$ & VLSN \\
\hline & GA1 & 3 & 10 & 847762.850 & 156755.347 & $(816505.912,879019.788)$ & VLSN \\
\hline & \multicolumn{7}{|c|}{ continued on the next page } \\
\hline
\end{tabular}


Table 2: Statistical Comparison of Experiments for $M=3$

\begin{tabular}{|c|c|c|c|c|c|c|c|}
\hline $\begin{array}{l}A_{1}: \\
\text { VLSN }\end{array}$ & $\begin{array}{l}A_{2}: \\
\text { Non- } \\
\text { VLSN }\end{array}$ & M & $\mathbf{n}$ & $\begin{array}{l}\text { Mean } \\
A_{1,2}\end{array}$ & Std. $D_{1,2}$ & 95\% C.I. & Winner \\
\hline & GA2 & 3 & 10 & 856478.770 & 169972.853 & $(822586.268,890371.272)$ & VLSN \\
\hline & Gurobi & 3 & 10 & -40711.660 & 32373.959 & $(-47167.011,-34256.309)$ & Non-VLSN \\
\hline \multirow{4}{*}{$\begin{array}{l}\text { Greedy- } \\
\text { VLSN }\end{array}$} & Greedy & 3 & 10 & 542307.910 & 320121.278 & $(156061.777,4065828346.104)$ & VLSN \\
\hline & GA1 & 3 & 10 & 760578.650 & 165832.273 & $(727511.777,793645.523)$ & VLSN \\
\hline & GA2 & 3 & 10 & 769294.570 & 196599.749 & $(730092.678,808496.462)$ & VLSN \\
\hline & Gurobi & 3 & 10 & -127895.860 & 87987.696 & $(-145440.563,-110351.157)$ & Non-VLSN \\
\hline \multirow{4}{*}{ VLSN1 } & Greedy & 3 & 20 & 585363.040 & 307288.252 & $(524089.915,646636.165)$ & VLSN \\
\hline & GA1 & 3 & 20 & 3823013.580 & 300278.765 & $(3763138.144,3882889.016)$ & VLSN \\
\hline & GA2 & 3 & 20 & 3835849.410 & 295563.710 & $(3776914.153,3894784.667)$ & VLSN \\
\hline & Gurobi & 3 & 20 & -236083.590 & 52816.299 & $(-246615.134,-225552.046)$ & Non-VLSN \\
\hline \multirow{4}{*}{ VLSNMS } & Greedy & 3 & 20 & 722671.110 & 303454.217 & $(662162.490,783179.730)$ & VLSN \\
\hline & GA1 & 3 & 20 & 3960321.650 & 298155.840 & $(3900869.524,4019773.776)$ & VLSN \\
\hline & GA2 & 3 & 20 & 3973157.480 & 301518.528 & $(3913034.836,4033280.124)$ & VLSN \\
\hline & Gurobi & 3 & 20 & -98775.520 & 20701.131 & $(-102903.315,-94647.725)$ & Non-VLSN \\
\hline \multirow{4}{*}{$\begin{array}{l}\text { Grid- } \\
\text { VLSN }\end{array}$} & Greedy & 3 & 20 & 672606.190 & 304057.858 & $(611977.204,733235.176)$ & VLSN \\
\hline & GA1 & 3 & 20 & 3910256.730 & 293280.628 & $(3851776.719,3968736.741)$ & VLSN \\
\hline & GA2 & 3 & 20 & 3923092.560 & 306168.609 & $(3862042.692,3984142.428)$ & VLSN \\
\hline & Gurobi & 3 & 20 & -148840.440 & 26144.873 & $(-154053.715,-143627.165)$ & Non-VLSN \\
\hline \multirow{4}{*}{$\begin{array}{l}\text { Greedy- } \\
\text { VLSN }\end{array}$} & Greedy & 3 & 20 & 640749.880 & 309907.226 & $(65603.813,18985277.328)$ & VLSN \\
\hline & GA1 & 3 & 20 & 3878400.420 & 299689.695 & $(3818642.444,3938158.396)$ & VLSN \\
\hline & GA2 & 3 & 20 & 3891236.250 & 310487.262 & $(3829325.244,3953147.256)$ & VLSN \\
\hline & Gurobi & 3 & 20 & -180696.750 & 56969.899 & $(-192056.520,-169336.980)$ & Non-VLSN \\
\hline \multirow{4}{*}{ VLSN1 } & Greedy & 3 & 30 & 547523.710 & 287548.220 & $(490186.738,604860.682)$ & VLSN \\
\hline & GA1 & 3 & 30 & 7434182.070 & 426287.497 & $(7349180.555,7519183.585)$ & VLSN \\
\hline & GA2 & 3 & 30 & 7499214.450 & 292365.595 & $(7440916.896,7557512.004)$ & VLSN \\
\hline & Gurobi & 3 & 30 & -272285.320 & 32905.022 & $(-278846.565,-265724.075)$ & Non-VLSN \\
\hline \multirow{4}{*}{ VLSNMS } & Greedy & 3 & 30 & 659756.550 & 291005.873 & $(601730.124,717782.976)$ & VLSN \\
\hline & GA1 & 3 & 30 & 7546414.910 & 428167.560 & $(7461038.512,7631791.308)$ & VLSN \\
\hline & GA2 & 3 & 30 & 7611447.290 & 290447.548 & $(7553532.193,7669362.387)$ & VLSN \\
\hline & Gurobi & 3 & 30 & -160052.480 & 15141.474 & $(-163071.682,-157033.278)$ & Non-VLSN \\
\hline \multirow{4}{*}{$\begin{array}{l}\text { Grid- } \\
\text { VLSN }\end{array}$} & Greedy & 3 & 30 & 619527.740 & 291727.276 & $(561357.466,677698.014)$ & VLSN \\
\hline & GA1 & 3 & 30 & 7506186.100 & 432202.493 & $(7420005.138,7592367.062)$ & VLSN \\
\hline & GA2 & 3 & 30 & 7571218.480 & 287537.072 & $(7513883.731,7628553.229)$ & VLSN \\
\hline & Gurobi & 3 & 30 & -200281.290 & 21595.991 & $(-204587.520,-195975.060)$ & Non-VLSN \\
\hline \multirow{4}{*}{$\begin{array}{l}\text { Greedy- } \\
\text { VLSN }\end{array}$} & Greedy & 3 & 30 & 623372.170 & 282526.737 & $(104743.167,536568.429)$ & VLSN \\
\hline & GA1 & 3 & 30 & 7510030.530 & 430923.987 & $(7424104.501,7595956.559)$ & VLSN \\
\hline & GA2 & 3 & 30 & 7575062.910 & 283967.037 & $(7518440.024,7631685.796)$ & VLSN \\
\hline & Gurobi & 3 & 30 & -196436.860 & 45216.148 & $(-205452.937,-187420.783)$ & Non-VLSN \\
\hline & Greedy & 3 & 40 & 632734.380 & 310776.474 & $(570765.706,694703.054)$ & VLSN \\
\hline \multicolumn{8}{|c|}{ VISN1 $\quad$ continued on the next page } \\
\hline
\end{tabular}


Table 2: Statistical Comparison of Experiments for $M=3$

\begin{tabular}{|c|c|c|c|c|c|c|c|}
\hline $\begin{array}{l}A_{1}: \\
\text { VLSN }\end{array}$ & $\begin{array}{l}A_{2}: \\
\text { Non- } \\
\text { VLSN }\end{array}$ & M & $\mathbf{n}$ & $\begin{array}{l}\text { Mean } \\
A_{1,2}\end{array}$ & Std. $D_{1,2}$ & 95\% C.I. & Winner \\
\hline & GA1 & 3 & 40 & 11370163.470 & 450560.750 & $(11280321.881,11460005.059)$ & VLSN \\
\hline & GA2 & 3 & 40 & 11159395.770 & 412442.699 & $(11077154.901,11241636.639)$ & VLSN \\
\hline & Gurobi & 3 & 40 & -301675.170 & 33257.627 & $(-308306.724,-295043.616)$ & Non-VLSN \\
\hline \multirow{4}{*}{ VLSNMS } & Greedy & 3 & 40 & 741892.330 & 308895.953 & $(680298.631,803486.029)$ & VLSN \\
\hline & GA1 & 3 & 40 & 11479321.420 & 445664.135 & $(11390456.213,11568186.627)$ & VLSN \\
\hline & GA2 & 3 & 40 & 11268553.720 & 410541.045 & $(11186692.040,11350415.400)$ & VLSN \\
\hline & Gurobi & 3 & 40 & -192517.220 & 13239.799 & $(-195157.229,-189877.211)$ & Non-VLSN \\
\hline \multirow{4}{*}{$\begin{array}{l}\text { Grid- } \\
\text { VLSN }\end{array}$} & Greedy & 3 & 40 & 702126.210 & 310744.835 & $(640163.844,764088.576)$ & VLSN \\
\hline & GA1 & 3 & 40 & 11439555.300 & 446812.411 & $(11350461.127,11528649.473)$ & VLSN \\
\hline & GA2 & 3 & 40 & 11228787.600 & 412326.091 & $(11146569.983,11311005.217)$ & VLSN \\
\hline & Gurobi & 3 & 40 & -232283.340 & 19798.391 & $(-236231.129,-228335.551)$ & Non-VLSN \\
\hline \multirow{4}{*}{$\begin{array}{l}\text { Greedy- } \\
\text { VLSN }\end{array}$} & Greedy & 3 & 40 & 738094.340 & 302753.314 & $(79306.429,4576228.208)$ & VLSN \\
\hline & GA1 & 3 & 40 & 11475523.430 & 450631.897 & $(11385667.654,11565379.206)$ & VLSN \\
\hline & GA2 & 3 & 40 & 11264755.730 & 411792.814 & $(11182644.448,11346867.012)$ & VLSN \\
\hline & Gurobi & 3 & 40 & -196315.210 & 42157.588 & $(-204721.412,-187909.008)$ & Non-VLSN \\
\hline \multirow{4}{*}{ VLSN1 } & Greedy & 3 & 50 & 532389.470 & 305910.186 & $\overline{(471391.131,593387.809)}$ & VLSN \\
\hline & GA1 & 3 & 50 & 15283969.450 & 570429.146 & $(15170226.162,15397712.738)$ & VLSN \\
\hline & GA2 & 3 & 50 & 15099111.000 & 503005.517 & $(14998811.950,15199410.050)$ & VLSN \\
\hline & Gurobi & 3 & 50 & -378638.790 & 39552.538 & $(-386525.546,-370752.034)$ & Non-VLSN \\
\hline \multirow{4}{*}{ VLSNMS } & Greedy & 3 & 50 & 663933.540 & 303157.072 & $(603484.171,724382.909)$ & VLSN \\
\hline & GA1 & 3 & 50 & 15415513.520 & 568004.680 & $(15302253.669,15528773.371)$ & VLSN \\
\hline & GA2 & 3 & 50 & 15230655.070 & 504040.517 & $(15130149.642,15331160.498)$ & VLSN \\
\hline & Gurobi & 3 & 50 & -247094.720 & 13459.298 & $(-249778.497,-244410.943)$ & Non-VLSN \\
\hline \multirow{4}{*}{$\begin{array}{l}\text { Grid- } \\
\text { VLSN }\end{array}$} & Greedy & 3 & 50 & 656987.910 & 307018.338 & $(595768.606,718207.214)$ & VLSN \\
\hline & GA1 & 3 & 50 & 15408567.890 & 568944.808 & $(15295120.578,15522015.202)$ & VLSN \\
\hline & GA2 & 3 & 50 & 15223709.440 & 503299.612 & $(15123351.748,15324067.132)$ & VLSN \\
\hline & Gurobi & 3 & 50 & -254040.350 & 14541.767 & $(-256939.971,-251140.729)$ & Non-VLSN \\
\hline \multirow{4}{*}{$\begin{array}{l}\text { Greedy- } \\
\text { VLSN }\end{array}$} & Greedy & 3 & 50 & 717863.920 & 302082.191 & $(132413.175,547247.574)$ & VLSN \\
\hline & GA1 & 3 & 50 & 15469443.900 & 569746.864 & $(15355836.659,15583051.141)$ & VLSN \\
\hline & GA2 & 3 & 50 & 15284585.450 & 505483.372 & $(15183792.317,15385378.583)$ & VLSN \\
\hline & Gurobi & 3 & 50 & -193164.340 & 32610.082 & $(-199666.774,-186661.906)$ & Non-VLSN \\
\hline \multirow{4}{*}{ VLSN1 } & Greedy & $\overline{3}$ & 100 & 432980.030 & 308760.169 & 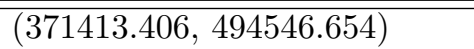 & "VLSN \\
\hline & GA1 & 3 & 100 & 36266744.820 & 641765.795 & $(36138777.040,36394712.600)$ & VLSN \\
\hline & GA2 & 3 & 100 & 35997235.880 & 725050.818 & $(35852661.107,36141810.653)$ & VLSN \\
\hline & Gurobi & 3 & 100 & -446354.930 & 37218.735 & $(-453776.327,-438933.533)$ & Non-VLSN \\
\hline \multirow{4}{*}{ VLSNMS } & Greedy & 3 & 100 & 572240.510 & 312392.330 & $(509949.635,634531.385)$ & VLSN \\
\hline & GA1 & 3 & 100 & 36406005.300 & 638373.264 & $(36278713.989,36533296.611)$ & VLSN \\
\hline & GA2 & 3 & 100 & 36136496.360 & 728320.389 & $(35991269.637,36281723.083)$ & VLSN \\
\hline & Gurobi & 3 & 100 & -307094.450 & 10890.716 & $(-309266.053,-304922.847)$ & Non-VLSN \\
\hline
\end{tabular}


Table 2: Statistical Comparison of Experiments for $M=3$

\begin{tabular}{|l|l|l|l|l|l|l|l|}
\hline $\begin{array}{l}A_{1}: \\
\text { VLSN }\end{array}$ & $\begin{array}{l}A_{2}: \\
\text { Non- } \\
\text { VLSN }\end{array}$ & $\mathbf{M}$ & $\mathbf{n}$ & $\begin{array}{l}\text { Mean } \\
A_{1,2}\end{array}$ & Std. $D_{1,2}$ & $\mathbf{9 5 \%}$ C.I. & Winner \\
\hline Grid- & Greedy & 3 & 100 & 594828.740 & 312548.849 & $(532506.655,657150.825)$ & \\
VLSN & GA1 & 3 & 100 & 36428593.530 & 638288.430 & $(36301319.135,36555867.925)$ & VLSN \\
& GA2 & 3 & 100 & 36159084.590 & 727803.960 & $(36013960.843,36304208.337)$ & VLSN \\
& Gurobi & 3 & 100 & -284506.220 & 10877.640 & $(-286675.216,-282337.224)$ & Non-VLSN \\
\hline & Greedy & 3 & 100 & 726941.570 & 309360.760 & $(134148.649,551656.943)$ & VLSN \\
GLSN & GA1 & 3 & 100 & 36560706.360 & 634202.488 & $(36434246.699,36687166.021)$ & VLSN \\
& GA2 & 3 & 100 & 36291197.420 & 726377.431 & $(36146358.122,36436036.718)$ & VLSN \\
& Gurobi & 3 & 100 & -152393.390 & 24995.680 & $(-157377.516,-147409.264)$ & Non-VLSN \\
\hline \hline
\end{tabular}

Table 3: Statistical Comparison of Experiments for $M=4$

\begin{tabular}{|c|c|c|c|c|c|c|c|}
\hline $\begin{array}{l}A_{1}: \\
\text { VLSN }\end{array}$ & $\begin{array}{l}A_{2}: \\
\text { Non- } \\
\text { VLSN }\end{array}$ & $\mathrm{M}$ & $\mathbf{n}$ & $\begin{array}{l}\text { Mean } \\
A_{1,2}\end{array}$ & Std. $D_{1,2}$ & 95\% C.I. & Winner \\
\hline \multirow{4}{*}{ VLSN1 } & Greedy & 4 & 4 & 420708.680 & 291983.871 & $(362487.241,478930.119)$ & VLSN \\
\hline & GA1 & 4 & 4 & -33290.710 & 64040.578 & $(-46060.369,-20521.051)$ & Non-VLSN \\
\hline & GA2 & 4 & 4 & -33290.710 & 64040.578 & $(-46060.369,-20521.051)$ & Non-VLSN \\
\hline & Gurobi & 4 & 4 & -33290.720 & 64040.574 & $(-46060.379,-20521.061)$ & Non-VLSN \\
\hline \multirow{4}{*}{ VLSNMS } & Greedy & 4 & 4 & 453999.390 & 273321.510 & $(399499.217,508499.563)$ & VLSN \\
\hline & GA1 & 4 & 4 & 0.000 & 0.000 & $(0.000,0.000)$ & Tie \\
\hline & GA2 & 4 & 4 & 0.000 & 0.000 & $(0.000,0.000)$ & Tie \\
\hline & Gurobi & 4 & 4 & -0.010 & 0.100 & $(-0.030,0.010)$ & None \\
\hline \multirow{4}{*}{$\begin{array}{l}\text { Grid- } \\
\text { VLSN }\end{array}$} & Greedy & 4 & 4 & 453999.390 & 273321.510 & $(399499.217,508499.563)$ & VLSN \\
\hline & GA1 & 4 & 4 & 0.000 & 0.000 & $(0.000,0.000)$ & Tie \\
\hline & GA2 & 4 & 4 & 0.000 & 0.000 & $(0.000,0.000)$ & Tie \\
\hline & Gurobi & 4 & 4 & -0.010 & 0.100 & $(-0.030,0.010)$ & None \\
\hline \multirow{4}{*}{$\begin{array}{l}\text { Greedy- } \\
\text { VLSN }\end{array}$} & Greedy & 4 & 4 & 342323.700 & 286661.924 & $(6464833.248,884074728781398.875)$ & VLSN \\
\hline & GA1 & 4 & 4 & -111675.690 & 118453.291 & $(-135295.217,-88056.163)$ & Non-VLSN \\
\hline & GA2 & 4 & 4 & -111675.690 & 118453.291 & $(-135295.217,-88056.163)$ & Non-VLSN \\
\hline & Gurobi & 4 & 4 & -111675.700 & 118453.283 & $(-135295.226,-88056.174)$ & Non-VLSN \\
\hline \multirow{4}{*}{ VLSN1 } & Greedy & 4 & 10 & 457756.410 & 303442.832 & $(397250.060,518262.760)$ & VLSN \\
\hline & GA1 & 4 & 10 & 849064.340 & 157667.201 & $(817625.579,880503.101)$ & VLSN \\
\hline & GA2 & 4 & 10 & 944836.200 & 122696.744 & $(920370.530,969301.870)$ & VLSN \\
\hline & Gurobi & 4 & 10 & -168329.980 & 35154.080 & $(-175339.686,-161320.274)$ & Non-VLSN \\
\hline \multirow{3}{*}{ VLSNMS } & Greedy & 4 & 10 & 580535.270 & 296673.117 & $(521378.798,639691.742)$ & VLSN \\
\hline & GA1 & 4 & 10 & 971843.200 & 145473.373 & $(942835.882,1000850.518)$ & VLSN \\
\hline & GA2 & 4 & 10 & 1067615.060 & 119273.555 & $(1043831.972,1091398.148)$ & VLSN \\
\hline
\end{tabular}


Table 3: Statistical Comparison of Experiments for $M=4$

\begin{tabular}{|c|c|c|c|c|c|c|c|}
\hline $\begin{array}{l}A_{1}: \\
\text { VLSN }\end{array}$ & $\begin{array}{l}A_{2}: \\
\text { Non- } \\
\text { VLSN }\end{array}$ & $\mathbf{M}$ & $\mathbf{n}$ & $\begin{array}{l}\text { Mean } \\
A_{1,2}\end{array}$ & Std. $D_{1,2}$ & 95\% C.I. & Winner \\
\hline & Gurobi & 4 & 10 & -45551.120 & 14835.867 & $(-48509.385,-42592.855)$ & Non-VLSN \\
\hline \multirow{4}{*}{$\begin{array}{l}\text { Grid- } \\
\text { VLSN }\end{array}$} & Greedy & 4 & 10 & 567159.630 & 297875.243 & $(507763.455,626555.805)$ & VLSN \\
\hline & GA1 & 4 & 10 & 958467.560 & 144629.304 & $(929628.549,987306.571)$ & VLSN \\
\hline & GA2 & 4 & 10 & 1054239.420 & 120087.033 & $(1030294.125,1078184.715)$ & VLSN \\
\hline & Gurobi & 4 & 10 & -58926.760 & 15813.094 & $(-62079.883,-55773.637)$ & Non-VLSN \\
\hline \multirow{4}{*}{$\begin{array}{l}\text { Greedy- } \\
\text { VLSN }\end{array}$} & Greedy & 4 & 10 & 471827.540 & 302512.696 & $(287030.494,48785145828.742)$ & VLSN \\
\hline & GA1 & 4 & 10 & 863135.470 & 154370.048 & $(832354.159,893916.781)$ & VLSN \\
\hline & GA2 & 4 & 10 & 958907.330 & 136883.720 & $(931612.784,986201.876)$ & VLSN \\
\hline & Gurobi & 4 & 10 & -154258.850 & 64071.598 & $(-167034.695,-141483.005)$ & Non-VLSN \\
\hline \multirow{4}{*}{ VLSN1 } & Greedy & 4 & 20 & 342618.340 & 295625.389 & $(283670.785,401565.895)$ & VLSN \\
\hline & GA1 & 4 & 20 & 3931114.510 & 337716.280 & $(3863774.052,3998454.968)$ & VLSN \\
\hline & GA2 & 4 & 20 & 3954816.290 & 282396.423 & $(3898506.584,4011125.996)$ & VLSN \\
\hline & Gurobi & 4 & 20 & -269337.000 & 32469.602 & $(-275811.423,-262862.577)$ & Non-VLSN \\
\hline \multirow{4}{*}{ VLSNMS } & Greedy & 4 & 20 & 481352.330 & 295531.385 & $(422423.519,540281.141)$ & VLSN \\
\hline & GA1 & 4 & 20 & 4069848.500 & 333945.221 & $(4003259.989,4136437.011)$ & VLSN \\
\hline & GA2 & 4 & 20 & 4093550.280 & 282354.181 & $(4037248.997,4149851.563)$ & VLSN \\
\hline & Gurobi & 4 & 20 & -130603.010 & 10977.705 & $(-132791.959,-128414.061)$ & Non-VLSN \\
\hline \multirow{4}{*}{$\begin{array}{l}\text { Grid- } \\
\text { VLSN }\end{array}$} & Greedy & 4 & 20 & 483696.310 & 294034.410 & $(425065.995,542326.625)$ & VLSN \\
\hline & GA1 & 4 & 20 & 4072192.480 & 333364.431 & $(4005719.778,4138665.182)$ & VLSN \\
\hline & GA2 & 4 & 20 & 4095894.260 & 282618.431 & $(4039540.285,4152248.235)$ & VLSN \\
\hline & Gurobi & 4 & 20 & -128259.030 & 10061.525 & $(-130265.293,-126252.767)$ & Non-VLSN \\
\hline \multirow{4}{*}{$\begin{array}{l}\text { Greedy- } \\
\text { VLSN }\end{array}$} & Greedy & 4 & 20 & 468652.290 & 291875.195 & $(76908.751,479624503.370)$ & VLSN \\
\hline & GA1 & 4 & 20 & 4057148.460 & 333773.486 & $(3990594.193,4123702.727)$ & VLSN \\
\hline & GA2 & 4 & 20 & 4080850.240 & 286310.078 & $(4023760.153,4137940.327)$ & VLSN \\
\hline & Gurobi & 4 & 20 & -143303.050 & 43853.404 & $(-152047.397,-134558.703)$ & Non-VLSN \\
\hline \multirow{4}{*}{ VLSN1 } & Greedy & 4 & 30 & 301879.240 & 299150.600 & $(242228.759,361529.721)$ & VLSN \\
\hline & GA1 & 4 & 30 & 7477163.470 & 324465.621 & $(7412465.187,7541861.753)$ & VLSN \\
\hline & GA2 & 4 & 30 & 7437036.990 & 369574.419 & $(7363344.035,7510729.945)$ & VLSN \\
\hline & Gurobi & 4 & 30 & -325354.810 & 37874.047 & $(-332906.876,-317802.744)$ & Non-VLSN \\
\hline \multirow{4}{*}{ VLSNMS } & Greedy & 4 & 30 & 457678.140 & 293766.388 & $(399101.268,516255.012)$ & VLSN \\
\hline & GA1 & 4 & 30 & 7632962.370 & 322434.530 & $(7568669.085,7697255.655)$ & VLSN \\
\hline & GA2 & 4 & 30 & 7592835.890 & 365974.022 & $(7519860.852,7665810.928)$ & VLSN \\
\hline & Gurobi & 4 & 30 & -169555.910 & 10206.322 & $(-171591.046,-167520.774)$ & Non-VLSN \\
\hline \multirow{4}{*}{$\begin{array}{l}\text { Grid- } \\
\text { VLSN }\end{array}$} & Greedy & 4 & 30 & 465655.330 & 295055.791 & $(406821.352,524489.308)$ & VLSN \\
\hline & GA1 & 4 & 30 & 7640939.560 & 323539.582 & $(7576425.928,7705453.192)$ & VLSN \\
\hline & GA2 & 4 & 30 & 7600813.080 & 364738.028 & $(7528084.499,7673541.661)$ & VLSN \\
\hline & Gurobi & 4 & 30 & -161578.720 & 10410.772 & $(-163654.623,-159502.817)$ & Non-VLSN \\
\hline \multirow[b]{2}{*}{ Greedy- } & Greedy & 4 & 30 & 503226.990 & 291500.030 & $(85368.942,509448384.965)$ & VLSN \\
\hline & GA1 & 4 & 30 & 7678511.220 & 324933.084 & $(7613719.725,7743302.715)$ & VLSN \\
\hline
\end{tabular}


Table 4 part of source data for $\mathrm{e}=40$

\begin{tabular}{|l|l|l|}
\hline$D_{1}$ & $D_{2}$ & $D_{3}$ \\
\hline Sigmund Freud & Sigmund Freud & SigmundxFreuv \\
\hline Graham Colditz & srShamNConditz & GrahaGOColditu \\
\hline Ronald C Kessler & RLnald C KmsslXr & pocalt C KEssler \\
\hline JoAnn E Manson & hoAnn AhYansan & JoAnniE Manson \\
\hline Shizuo Akira & ShMzuI Tkila & zhizuoyAkCra \\
\hline
\end{tabular}

Table 3: Statistical Comparison of Experiments for $M=4$

\begin{tabular}{|l|l|l|l|l|l|l|l|}
\hline $\begin{array}{l}A_{1}: \\
\text { VLSN }\end{array}$ & $\begin{array}{l}A_{2}: \\
\text { Non- } \\
\text { VLSN }\end{array}$ & $\mathbf{M}$ & $\mathbf{n}$ & $\begin{array}{l}\text { Mean } \\
A_{1,2}\end{array}$ & Std. $D_{1,2}$ & $\mathbf{9 5 \%}$ C.I. & Winner \\
\hline & GA2 & 4 & 30 & 7638384.740 & 364695.841 & $(7565664.571,7711104.909)$ & VLSN \\
& Gurobi & 4 & 30 & -124007.060 & 35641.249 & $(-131113.907,-116900.213)$ & Non-VLSN \\
\hline \hline
\end{tabular}

Column $A_{1}$ : VLSN contains a name of VLSN version. Column $A_{2}$ : NonVLSN lists a name of non-VLSN algorithm that is compared with the VLSN search in the same row. Columns $A_{1,2}, D_{1,2}$, and $\mathbf{9 5 \%}$ C.I. display the sample mean, the sample standard deviation, and the $95 \%$ confidence interval for $y_{1}-y_{2}$.

\subsection{Illustration of application to multipartite entity resolution problem}

Current experiment presents illustration of application of proposed algorithms to multipartite entity resolution problem. Experiment settings and results are displayed in table 5 . We have collected list of 2610 highly cited researcher names from (http://www.webometrics.info/en/node/58) and selected subset of size $\mathbf{N}$ of their names. Next, we created three identical datasets $D_{1}, D_{2}$, and $D_{3}$. Column e denotes error: probability under which random letter of any item in $D_{1}$ or $D_{2}$ would be changed to random letter. Part of distorted dataset is shown in table 4. We have generated input matrix as per formulation 2 , using trigram similarity as similarity function, and performed greedy search as in algorithm 1, and then improved it using VLSN with greedy start (alg. 2). Algorithms return solutions as set of 3 -tuples $\left(\left(d_{1}^{1}, d_{2}^{1}, d_{3}^{1}\right), \ldots,\left(d_{1}^{N}, d_{2}^{N}, d_{3}^{N}\right)\right)$, meaning that $d_{1}^{1}, d_{2}^{1}$, and $d_{3}^{1}$ denote the same entity. We define 3 -tuple as correctly matched, if all its elements correspond to the record for the same entity, otherwise the 3-tuple is matched incorrectly. We define recall of the algorithm as the ratio of correctly matched 3-tuples to the total number of 3-tuples. Columns $\mathbf{R}(\mathbf{G})$ and $\mathbf{R}\left(\mathbf{V}_{-} \mathbf{G}\right)$ denote recall for greedy and for VLSNgreedy algorithms respectively. Column $|V|$ denotes number of nodes in the resulting graph. Results of this experiment show that recall for VLSN search improves over greedy search. 
Table 5 Results of application of MAP to multipartite entity resolution problem

\begin{tabular}{|l|l|l|l|l|l|l|l|}
\hline$\#$ & $\mathbf{N}$ & $\mathbf{e}$ & $\mathbf{G}$ & $\mathbf{V} \_\mathbf{G}$ & $|V|$ & $\mathbf{R}(\mathbf{G})$ & $\mathbf{R}\left(\mathbf{V} \_\mathbf{G}\right)$ \\
\hline 1 & 10 & 0 & 10 & 10 & 1 & 1.0 & 1.0 \\
\hline 2 & 10 & 50 & 6 & 10 & 4 & 0.6 & 1.0 \\
\hline 3 & 20 & 20 & 20 & 20 & 1 & 1.0 & 1.0 \\
\hline 4 & 20 & 30 & 17 & 20 & 2 & 0.85 & 1.0 \\
\hline 5 & 40 & 20 & 40 & 40 & 1 & 1.0 & 1.0 \\
\hline 6 & 20 & 50 & 16 & 20 & 4 & 0.8 & 1.0 \\
\hline 7 & 50 & 10 & 50 & 50 & 1 & 1.0 & 1.0 \\
\hline 8 & 10 & 20 & 10 & 10 & 1 & 1.0 & 1.0 \\
\hline 9 & 20 & 10 & 20 & 20 & 1 & 1.0 & 1.0 \\
\hline 10 & 20 & 40 & 20 & 20 & 1 & 1.0 & 1.0 \\
\hline 11 & 10 & 40 & 6 & 8 & 4 & 0.6 & 0.8 \\
\hline 12 & 20 & 0 & 20 & 20 & 1 & 1.0 & 1.0 \\
\hline 13 & 10 & 30 & 8 & 10 & 2 & 0.8 & 1.0 \\
\hline 14 & 10 & 10 & 10 & 10 & 1 & 1.0 & 1.0 \\
\hline 15 & 40 & 10 & 40 & 40 & 1 & 1.0 & 1.0 \\
\hline 16 & 50 & 20 & 48 & 48 & 2 & 0.96 & 0.96 \\
\hline 17 & 40 & 30 & 31 & 38 & 4 & 0.775 & 0.95 \\
\hline 18 & 40 & 40 & 30 & 37 & 7 & 0.75 & 0.925 \\
\hline 19 & 100 & 10 & 100 & 100 & 1 & 1.0 & 1.0 \\
\hline 20 & 50 & 30 & 36 & 50 & 5 & 0.72 & 1.0 \\
\hline 21 & 100 & 20 & 96 & 98 & 2 & 0.96 & 0.98 \\
\hline 22 & 50 & 40 & 38 & 46 & 13 & 0.76 & 0.92 \\
\hline 23 & 40 & 50 & 22 & 26 & 25 & 0.55 & 0.65 \\
\hline 24 & 50 & 50 & 20 & 28 & 13 & 0.4 & 0.56 \\
\hline 25 & 100 & 30 & 72 & 96 & 13 & 0.72 & 0.96 \\
\hline 26 & 300 & 10 & 289 & 300 & 4 & 0.96 & 1.0 \\
\hline 27 & 100 & 40 & 54 & 71 & 35 & 0.54 & 0.71 \\
\hline 28 & 100 & 50 & 31 & 47 & 83 & 0.31 & 0.47 \\
\hline 29 & 300 & 20 & 269 & 300 & 9 & 0.89 & 1.0 \\
\hline 30 & 300 & 30 & 207 & 276 & 20 & 0.69 & 0.92 \\
\hline 31 & 300 & 40 & 131 & 201 & 82 & 0.43 & 0.67 \\
\hline & & & & & & & \\
\hline
\end{tabular}

\section{Conclusions and further research}

Current paper describes mathematical derivation of multidimensional assignment problem and its application to multipartite entity matching. Exact solution of MAP is NP-hard; in current paper we present two heuristic approaches to solve the problem. Because the new application to multipartite entity resolution requires solving problems with large cardinality, exact approaches become computationally infeasible. Heuristic and metaheuristic approaches are more scalable; but they require intelligent strategies for exploration of vast solution set. Therefore, understanding which multistart approaches should be used in exploration phase of a search become important. We add to this underinvestigated area by studying three alternative ways to restart VLSN search algorithm, including random restart, grid-based restart and greedy restart.

Limitation of current approach is necessity to have entire matrix with comparison filled with values; it means, that all elements of all datasets should be compared to each other. Due to practical limitations that cannot be performed 
for large datasets. We aim at resolving this limitation by reformulating MAP taking into account blocking, or Top-K similarity queries. Next, in current experiments matrices are generated using uniform random distribution. We believe that in real data, when matrices are constructed using comparisons, distributions will be other than uniform random, and it would lead to different properties of solutions.

\section{Acknowledgment}

A. Kammerdiner was supported by the AFRL (National Research Council Fellowship). The work of A. Semenov was funded in part by the AFRL European Office of Aerospace Research and Development (Grant FA9550-17-1-0030, University of Jyvaskyla, Finland).

\section{Data availability statement}

All data generated or analysed during this study are included in this published article.

\section{References}

1. E. Adar, M. Hurst, T. Finin, N. S. Glance, N. Nicolov, and B. L. Tseng, editors. Proceedings of the Third International Conference on Weblogs and Social Media, ICWSM 2009, San Jose, California, USA, May 17-20, 2009. The AAAI Press, 2009.

2. C. Arbib, D. Pacciarelli, and S. Smriglio. A three-dimensional matching model for perishable production scheduling. Discrete Applied Mathematics, 92(1):1-15, 1999

3. E. Balas and P. R. Landweer. Traffic assignment in communication satellites. Operations Research Letters, 2(4):141-147, 1983.

4. O. Benjelloun, H. Garcia-Molina, D. Menestrina, Q. Su, S. E. Whang, and J. Widom. Swoosh: A Generic Approach to Entity Resolution. The VLDB Journal, 18(1):255-276, Jan. 2009

5. D. Brizan and A. Tansel. A Survey of Entity Resolution and Record Linkage Methodologies. Communications of the IIMA, 6(3):41-50, 2006.

6. R. Burkard, M. Dell'Amico, and S. Martello. Assignment problems, revised reprint, volume 106. Siam, 2012.

7. R. E. Burkard and E. Cela. Linear assignment problems and extensions. In Handbook of combinatorial optimization, pages 75-149. Springer, 1999

8. X. Chu, I. F. Ilyas, and P. Koutris. Distributed data deduplication. Proc. VLDB Endow., 9(11):864-875, July 2016.

9. Y. Crama, O. E. Flippo, J. Van de Klundert, and F. C. Spieksma. The assembly of printed circuit boards: A case with multiple machines and multiple board types. European journal of operational research, 98(3):457-472, 1997.

10. Y. Crama, A. G. Oerlemans, and F. C. Spieksma. Production planning in automated manufacturing. Springer Science \& Business Media, 2012.

11. A. K. Elmagarmid, P. G. Ipeirotis, and V. S. Verykios. Duplicate Record Detection A Survey. IEEE Transactions on Knowledge and Data Engineering, 19(1):1-16, Jan. 2007.

12. D. Firmani, B. Saha, and D. Srivastava. Online Entity Resolution Using an Oracle. Proc. VLDB Endow., 9(5):384-395, Jan. 2016. 
13. A. Frieze and J. Yadegar. An algorithm for solving 3-dimensional assignment problems with application to scheduling a teaching practice. Journal of the operational research society, 32(11):989-995, 1981.

14. M. R. Garey and D. S. Johnson. Computers and intractability, volume 174. freeman San Francisco, 1979.

15. K. C. Gilbert and R. B. Hofstra. An algorithm for a class of three-dimensional assignment problems arising in scheduling applications. IIE transactions, 19(1):29-33, 1987.

16. K. C. Gilbert and R. B. Hofstra. Multidimensional assignment problems. Decision Sciences, 19(2):306-321, 1988

17. C. Gokhale, S. Das, A. Doan, J. F. Naughton, N. Rampalli, J. Shavlik, and X. Zhu. Corleone: Hands-off crowdsourcing for entity matching. In Proceedings of the 2014 ACM SIGMOD International Conference on Management of Data, SIGMOD '14, pages 601612, New York, NY, USA, 2014. ACM.

18. S. Guo, X. L. Dong, D. Srivastava, and R. Zajac. Record linkage with uniqueness constraints and erroneous values. Proc. VLDB Endow., 3(1-2):417-428, Sept. 2010.

19. G. Gutin, B. Goldengorin, and J. Huang. Worst case analysis of max-regret, greedy and other heuristics for multidimensional assignment and traveling salesman problems. Journal of Heuristics, 14(2):169-181, 2008.

20. J. He, H. Liu, R. Y. K. Lau, and J. He. Relationship Identification Across Heterogeneous Online Social Networks. Computational Intelligence, Jan. 2016.

21. D. Helbing and S. Balietti. From social data mining to forecasting socio-economic crises. The European Physical Journal Special Topics, 195(1):3, 2011.

22. A. Hilton. The reconstruction of latin squares with applications to school timetabling and to experimental design. In Combinatorial Optimization II, pages 68-77. Springer, 1980.

23. P. Jain, P. Kumaraguru, and A. Joshi. @i seek 'fb.me': Identifying users across multiple online social networks. In Proceedings of the 22Nd International Conference on World Wide Web, WWW'13 Companion, pages 1259-1268, New York, NY, USA, 2013. ACM.

24. A. Kammerdiner. Ranking risk exposures for situational surveillance of falls with sensors. Operations Research for Health Care, 7:132-137, 2015.

25. A. Kammerdiner, P. Krokhmal, and P. Pardalos. Characteristics of the distribution of hamming distance values between multidimensional assignment problem solutions. Advances in Cooperative Control and Optimization, pages 339-352, 2007.

26. A. Kammerdiner and C. F. Vaughan. Very large-scale neighborhood search for the multidimensional assignment problem. In S. Butenko, P. M. Pardalos, and V. Shylo, editors, Optimization Methods and Applications. Springer, 2017.

27. A. R. Kammerdiner. Multidimensional assignment problem multidimensional assignment problem. In Encyclopedia of Optimization, pages 2396-2402. Springer, 2008.

28. A. R. Kammerdiner and A. N. Guererro. Data-driven combinatorial optimization for sensor-based assessment of near falls. Annals of Operations Research, 276(1-2):137-153, 2019.

29. A. R. Kammerdiner, A. Mucherino, and P. M. Pardalos. Application of monkey search meta-heuristic to solving instances of the multidimensional assignment problem. In Optimization and Cooperative Control Strategies, pages 385-397. Springer, 2009.

30. D. Karapetyan and G. Gutin. Local search heuristics for the multidimensional assignment problem. Journal of Heuristics, 17(3):201-249, 2011.

31. R. M. Karp. Reducibility among combinatorial problems. In Complexity of computer computations, pages 85-103. Springer, 1972.

32. H. Köpcke, A. Thor, and E. Rahm. Evaluation of entity resolution approaches on real-world match problems. Proc. VLDB Endow., 3(1-2):484-493, Sept. 2010.

33. P. A. Krokhmal. On optimality of a polynomial algorithm for random linear multidimensional assignment problem. Optimization Letters, 5(1):153-164, 2011.

34. F. Li, M. L. Lee, W. Hsu, and W.-C. Tan. Linking temporal records for profiling entities. In Proceedings of the 2015 ACM SIGMOD International Conference on Management of Data, SIGMOD '15, pages 593-605, New York, NY, USA, 2015. ACM.

35. D. M. Nguyen, H. A. Le Thi, and T. P. Dinh. Solving the multidimensional assignment problem by a cross-entropy method. Journal of Combinatorial Optimization, 27(4):808823, 2014. 
36. G. Papadakis, J. Svirsky, A. Gal, and T. Palpanas. Comparative analysis of approximate blocking techniques for entity resolution. Proc. VLDB Endow., 9(9):684-695, May 2016.

37. E. L. Pasiliao Jr. Local neighborhoods for the multidimensional assignment problem. In Dynamics of information systems, pages 353-371. Springer, 2010.

38. W. P. Pierskalla. The tri-substitution method for the three-dimensional assignment problem. CORS Journal, 5:71-81, 1967.

39. W. P. Pierskalla. Letter to the editor - the multidimensional assignment problem. $O p$ erations Research, 16(2):422-431, 1968.

40. A. Poore, N. Rijavec, M. Liggins, and V. Vannicola. Data association problems posed as multidimensional assignment problems: problem formulation. In Optical Engineering and Photonics in Aerospace Sensing, pages 552-563. International Society for Optics and Photonics, 1993.

41. S. Puglisi, D. Rebollo-Monedero, and J. Forné. On Web user tracking: How thirdparty http requests track users' browsing patterns for personalised advertising. In 2016 Mediterranean Ad Hoc Networking Workshop (Med-Hoc-Net), pages 1-6, June 2016.

42. J.-F. Pusztaszeri. The nonlinear assignment problem in experimental high energy physics. In Nonlinear Assignment Problems, pages 55-89. Springer, 2000.

43. J.-F. Pusztaszeri, P. E. Rensing, and T. M. Liebling. Tracking elementary particles near their primary vertex: a combinatorial approach. Journal of Global Optimization, 9(1):41-64, 1996.

44. C. Riederer, Y. Kim, A. Chaintreau, N. Korula, and S. Lattanzi. Linking Users Across Domains with Location Data: Theory and Validation. In Proceedings of the 25th International Conference on World Wide Web, WWW'16, pages 707-719, Republic and Canton of Geneva, Switzerland, 2016. International World Wide Web Conferences Steering Committee.

45. T. Sagi, A. Gal, O. Barkol, R. Bergman, and A. Avram. Multi-source uncertain entity resolution at yad vashem: Transforming holocaust victim reports into people. In Proceedings of the 2016 International Conference on Management of Data, SIGMOD '16, pages 807-819, New York, NY, USA, 2016. ACM.

46. A. Semenov and J. Veijalainen. A modelling framework for social media monitoring. International Journal of Web Engineering and Technology, 8(3):217-249, 2013. PMID: 57226.

47. K. Shu, S. Wang, J. Tang, R. Zafarani, and H. Liu. User identity linkage across online social netwroks: A review. In To appear in SIGKDD Explorations, To appear in SIGKDD Explorations, 2016.

48. R. M. Silva, M. G. Resende, and P. M. Pardalos. Finding multiple roots of a boxconstrained system of nonlinear equations with a biased random-key genetic algorithm. J. of Global Optimization, 60(2):289-306, Oct. 2014.

49. C. Vogiatzis, E. L. Pasiliao, and P. M. Pardalos. Graph partitions for the multidimensional assignment problem. Computational Optimization and Applications, 58(1):205$224,2014$.

50. J. Wang, T. Kraska, M. J. Franklin, and J. Feng. CrowdER: Crowdsourcing Entity Resolution. Proc. VLDB Endow., 5(11):1483-1494, July 2012.

51. T. Ye and H. W. Lauw. Structural constraints for multipartite entity resolution with markov logic network. In Proceedings of the 24th ACM International on Conference on Information and Knowledge Management, CIKM '15, pages 1691-1694, New York, NY, USA, 2015. ACM.

52. D. Zhang, B. I. P. Rubinstein, and J. Gemmell. Principled Graph Matching Algorithms for Integrating Multiple Data Sources. IEEE Transactions on Knowledge and Data Engineering, 27(10):2784-2796, Oct. 2015.

53. J. Zhang and P. S. Yu. Multiple Anonymized Social Networks Alignment. In 2015 IEEE International Conference on Data Mining, pages 599-608, Nov. 2015.

54. X. Zhou, X. Liang, H. Zhang, and Y. Ma. Cross-platform identification of anonymous identical users in multiple social media networks. IEEE Trans. on Knowl. and Data Eng., 28(2):411-424, Feb. 2016.

55. X.-H. ZHOU and S. Gao. Confidence intervals for the log-normal mean. Statistics in medicine, 16(7):783-790, 1997. 